\title{
Influencia del fenómeno El Niño en la región occidental de Cuba y su impacto en la pesquería de langosta (Panulirus argus) del golfo de Batabanó
}

\author{
Benigno Hernández de la T. y Rafael Puga M. \\ Centro de Investigaciones Pesqueras \\ 5ta. Ave. y 248 Barlovento, Playa, Ciudad de La Habana, Cuba
}

\begin{abstract}
RESUMEN. Se analizan variables y eventos hidrometeorológicos que muestran in situ la influencia que ejerce el fenómeno El Niño en la región occidental de Cuba. En particular se demuestra, estadísticamente por medio del método de análisis de épocas superpuestas, que las capturas totales de langosta (Panulirus argus) en el golfo de Batabanó son altas en años de incidencia del fenómeno y bajas en los años -1 y +1 . El grado de asociación obtenido, después de diez mil intentos por el método de Monte Carlo, arrojó una probabilidad $\mathrm{P}=0,001$ para la captura total, $\mathrm{P}=0,0038$ para la del levante de la veda y $\mathrm{P}=0,0226$ para el período de migraciones masivas del recurso.

Palabras claves: El Niño, langosta, Panulirus argus, épocas superpuestas, golfo de Batabanó, Cuba.

\section{The influence of the El Niño phenomenon over the western region of Cuba and upon the lobster fishery (Panulirus argus) in the gulf of Batabanó}

\begin{abstract}
Variables and hydro-meteorological events showing the in situ influence of the El Niño phenomenon aver the western region of Cuba are analyzed. It is statistically shown, using the overlaping epochs analysis method, that the total catch of lobsters (Panulirus argus) in the gulf of Batabanó are high in El Niño years and low in the years +1 and 1. After ten thousand iterations by the Monte Carlo method, the degree of association for the total catch gave a probability of $\mathrm{P}=0.001 \mathrm{P}=0.0038$ for the terrnination of the closed season, and $\mathrm{P}=0.0226$ for the period of massive migration.
\end{abstract}

Key words: El Niño, lobster, Panulirus argus, overlap, gulf of Batabanó, Cuba.

\section{INTRODUCCION}

Mucho se ha escrito desde que en 1972-73 aguas calientes irrumpieron en las costas de Perú y Chile, como preludio del desastre de la pesquería de anchoveta en esas costas, asociadas al evento El Niño. Lo mismo sucede años después cuando el fenómeno reaparece en 1976-77 y 1982-83, acentuando aún más el colapso de esa pesquería (Caviedes, 1975; Arntz, 1984; Quinn y Neal, 1989; Glantz, 1991; Yáñez, 1991).

Este fenómeno de interacción océano-atmósfera ha desatado en sus últimas apariciones una estela de desastres en la pesca y economía de diversos países, razón por la cual muchos consideran de catas- trófica solamente la aparición de un evento pronosticado como débil.

El ENOS (El Niño/Oscilación del Sur) es el resultado de un vasto y complejo sistema de oscilaciones que se desarrolla en el hemisferio sur, donde interactúan la atmósfera y el océano, causando importantes perturbaciones hidroclimatológicas en diversas zonas, como por ejemplo en los regímenes de lluvia en áreas usualmente desérticas y sequías en zonas lluviosas.

En Cuba este fenómeno afecta directamente la región occidental (Hernández, 1992), con fuertes gradientes en distintas variables y eventos meteorológicos y oceánicos, por lo que puede afectar o favorecer el régimen pesquero de la plataforma 
suroccidental o golfo de Batabanó, donde se han desarrollado importantes pesquerías, entre ellas la de langosta (Panulirus argus).

Las labores extractivas en esta pesquería se desarrollan entre el 1 de junio y el 28 de febrero del año siguiente. En la explotación del recurso se presentan dos períodos fundamentales de captura: el que comienza al finalizar el período de veda, llamado «levante de veda», y el de migración masiva del recurso, conocido como «recalo». Esta última representa un porcentaje importante dentro del total nacional, y se asocia principalmente a los disturbios invernal es, como frentes fríos, bajas extratropicales y huracanes que se desarrollan en el golfo de México y Mar Caribe (Hernández, 1990; García et al., 1991).
El presente trabajo tiene como objetivo mostrar el efecto que ejercen los episodios El Niño en la región occidental de Cuba y demostrar que éstos influyen en la pesquería de langosta. Por la extensión de los datos biológicos y la seriedad en la secuencia de las interrelaciones con los parámetros abióticos, se considera ésta como la primera investigación que comprueba estadísticamente la asociación entre el fenómeno de El Niño con especies marinas cubanas.

\section{MATERIALES Y METODOS}

En el presente trabajo se utilizan las anomalías de la presión atmosférica, lluvia y temperatura del aire

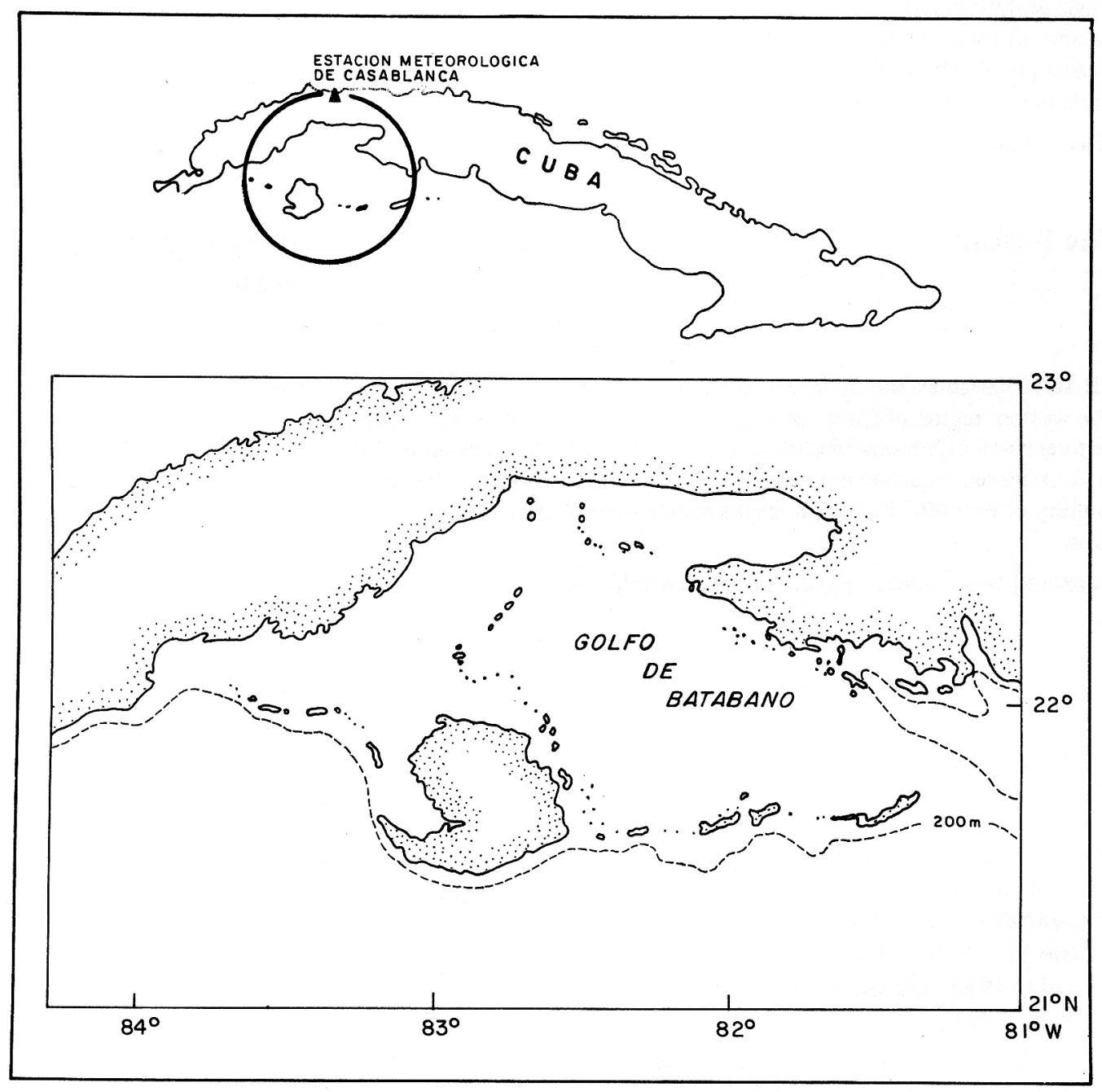

Figura l. Ubicación de la estación meteorológica de Casablanca y del golfo de Batabanó en la región occidental de Cuba. 
correspondientes a los meses invernales (octubremarzo), período poco lluvioso. Los datos analizados, desde 1923 hasta la temporada 1992-93, provienen de la estación meteorológica de Casablanca (Fig. 1). Esta se sitúa en los $23^{\circ} 10^{\prime} \mathrm{N}$ y $82^{\circ} 21^{\prime} \mathrm{W}$, a $50 \mathrm{~m}$ de altura sobre el nivel del mar en la entrada de la Bahía de La Habana, con la característica de ser una estación de primer orden, es decir, bien ubicada para registrar variaciones del clima en la región occidental. Las anomalías de las variables fueron obtenidas a partir de una media 0 y desviación estándar 1. Los registros de perturbaciones tropicales, como huracanes y frentes, se tomaron del Departamento de Infonnación y Sinóptica del Instituto de Meteorología y de la cronología de frentes fríos (Rodríguez, 1982).

Las anomalías de la temperatura superficial del mar (TSM) de 1970 a 1993, fueron proporcionadas por el Dr. Reynolds de la NOAA (com. pers.) para el cuadrante de Cuba $\left(19^{\circ}-24^{\circ} \mathrm{N}\right.$ y $\left.75^{\circ}-85^{\circ} \mathrm{W}\right)$. Del Instituto Cubano de Hidrografía se recuperaron las anomalías del nivel medio del mar (NMM) en la estación mareo gráfica de Casilda $\left(21^{\circ} 40^{\prime} \mathrm{N}\right.$; $79^{\circ} 59^{\prime} \mathrm{W}$ ), expresadas en su promedio móvil de 12 meses.

Las anomalías del Indice de Oscilación Sur (IOS) de 1964 a 1993, y de la TSM en la región Niño 3 de 1970 a 1993, fueron proporcionadas respectivamente por el Dr. Kousky y el Dr. Enfield de la NOAA (com. pers.). Los eventos ENOS que se estudian son los de 1965-66, 1969-70, 1972-73, 1976-77, 1982-83, 1987-88 y 1991-92; después de este último evento no se habría retornado aún a una situación nonnal. Los estudios y resultados relacionados con este fenómeno, así como datos e interpretaciones, se obtuvieron de la consulta de diversos trabajos de especialistas dedicados al tema.

Los registros mensuales del desembarque de lan gosta fueron obtenidos a partir de los tres Combinados Pesqueros Industriales que operan en el golfo de Batabanó desde 1964 hasta 1992-93, expresados en miles de toneladas (t). En esta región se desarrolla la pesquería más importante a nivel nacional, aportando alrededor de $7.000 \mathrm{t}$ anuales, es decir, aproximadamente el $60 \%$ de la captura total nacional (Cruz et al., 1992a). No se trabajó con la CPUE, puesto que esta pesquería utiliza dos artes de pesca representativos de cada período, y no se ha llegado a un consenso sobre la estandarización de los esfuerzos realizados con dichas artes para obtener un rendimiento por temporada de pesca.

Los datos biológicos fueron procesados con el paquete de programas SISLAN (Sotomayor y Cruz, 1991), estableciendo los dos principales períodos de captura que componen la temporada de pesca: $<<1$ vante de veda» (junio-septiembre) y «recalo» (octubrefebrero).

El método que se utilizó para relacionar estadísticamente las variaciones de la pesquería con los eventos ENOS fue el análisis de épocas superpuestas (Panofski y Brier, 1965; Haurtwitz y Brier, 1981; Prager y Hoening, 1989, 1992). Este es una técnica no-paramétrica, que puede ser usada para probar significancias estadísticas de asociaciones entre eventos discretos autocorrelacionados (fenómenos ambientales extremos, como El Niño, huracanes, frentes, etc.) y otros sucesos. La prueba es análoga al t de Student (R), que compara la media obtenida en cada evento clave con los años contiguos. Una vez obtenido este número, se simula por el método de Monte Carlo, brindando la probabilidad real que este análisis tiene de no ser aleatorio, es decir, más dependiente del evento clave en cuestión. Como el análisis no requiere de asunciones iniciales, su aplicación directa es válida y adecuada. El programa original en FORTRAN (Hoening et al., 1989), que pennite computar las pruebas estadísticas mediante simulación aleatoria por el método de Monte Carlo, se compiló en $\mathrm{C}$ y se adaptó a microcomputadoras IBM compatibles (Alfonso, 1992).

\section{RESULTADOS Y DISCUSION}

\section{Mecanismo de acción de El Niño en la región occi- dental de Cuba}

Existe una amplia literatura sobre el fenómeno ENOS, contemplando aspectos teóricos (Cane et al., 1986), observacionales (Rasmusson, 1991) y de pronóstico (Zebiak y Cane, 1987). Tambien existen trabajos sobre la influencia que ejerce el fenómeno sobre la economía de diversos países (Nicholls, 1986), y específicamente sobre las capturas de especies marinas de interés comercial a nivel mundial (Pauly, 1987; Yáñez, 1989; White y Downton, 1991).

El ENOS es considerado actualmente como la señal dominante del clima global para escalas de tiempo que oscilan desde meses hasta algunos años 
(Enfield, 1987; Galindo y Centeno, 1989). El Niño es la componente oceánica de ENOS y se manifiesta a través de los cambios en las anomalías de la TSM en regiones del Pacífico Tropical (Trenberth, 1991). Mientras que la Oscilación del Sur es tomada generalmente por la comunidad científica internacional como la señal avisora del estado del fenómeno en la región del Pacífico y proviene de las diferencias de presiones a nivel del mar entre las estaciones meteorológicas de Darwin y Tahití (Climate Analysis Center, 1993; BMA, 1992).

El ENOS despliega una decena de patrones teleconectivos a través de las oscilaciones de la corriente en chorro subtropical, la que durante el invierno del hemisferio norte se intensifica de manera atípica, para acentuar situaciones meteorológicas extremas en distintas regiones del mundo.

Según White y Downton (1991), varios autores han demostrado la incidencia durante el invierno del hemisferio norte de tres patrones que afectan la circulación en el sureste de Estados Unidos, el golfo de México y la región occidental de Cuba. Estos son el IOS (Ropelewski y Halpert, 1986; Bradley et al., 1987), la Oscilación del Atlántico Norte (van Loon y Rogers, 1978; Meehl, 1978), y el patrón del Pacífico de Norte América (PNA) (Dickson y Namías, 1976; Horel y Wallace, 1981).

La Fig. 2 muestra el esquema de teleconexiones observadas en la atmósfera según Rasmusson y Wallace (1983). La Fig. 2a ilustra las anomalías típicas de la circulación en la iropósfera alta sobre el Pacífico desde diciembre a febrero, compuesta por varios fenómenos ENOS anteriores al de 1982-83. Estos incluyen un par de anticiclones hacia el norte y sur del Ecuador, a favor y en contra de las manecillas del reloj respectivamente. También se observa la tripleta circulatoria de centros giratorios ciclónicos y anticiclónicos del Pacífico norte, Canadá, sureste de Estados Unidos y Atlántico occidental, identificada por Horel y Wallace (1981) como patrón teleconectivo PNA (Rasmusson, 1991). La extensión hacia el E de la nubosidad y la lluvia asociada al evento 1982-83, se muestra en la Fig. 2b. Esta señala el fortalecimiento enorme que tuvieron los anticiclones ecuatoriales en ambos hemisferios; los que se extendieron mucho más hacia el E, y la extensión atípica de la tripleta de altas y bajas en el hemisferio norte (Rasmusson, 1991).

Las presiones medias mensuales de la corriente en chorro subtropical en la región de las Aleutianas,
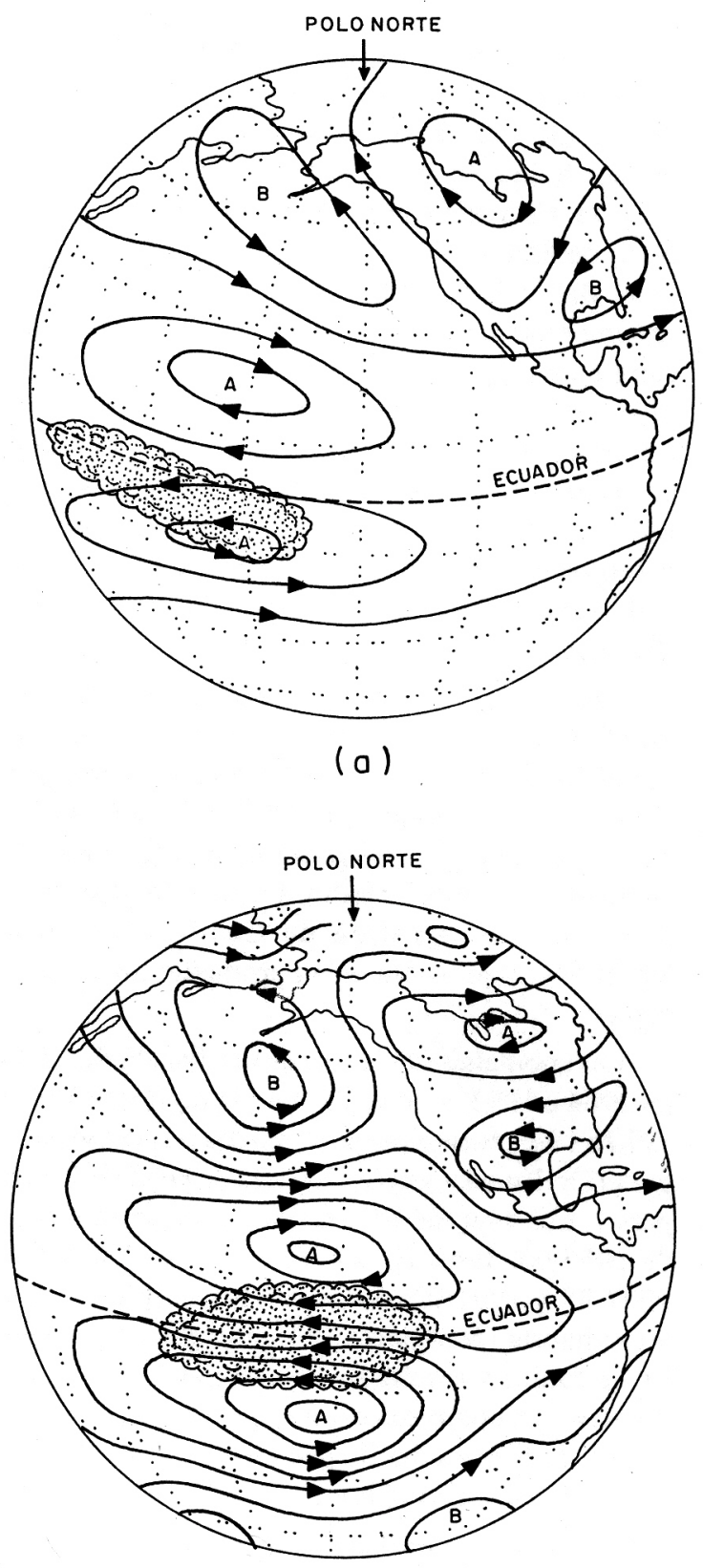

(b)

Figura 2. Teleconexiones observadas en la tropósfera alta: a) anomalías típicas de la circulación sobre el Pacífico durante el invierno del hemisferio norte, compuesta por varios eventos ENOS anteriores al de 1982-83; y b) durante el episodio caliente de 1982-83 (Rasmusson y Wallace, 1983).

en el golfo de Alaska, alcanzaron durante 1982-83 valores bajos cercanos al record; en tanto que los rumbos de las tormentas que penetraron en América del 
Norte, provenientes del Pacífico, fueron desplazadas por momentos cientos de millas hacia el $\mathrm{SE}$, trayendo como consecuencia vientos y mareas destructivas hacia la costa de California. La extensión hacia el E y el desplazamiento hacia el S de la corriente en chorro en Baja California y el golfo de México, se asoció con períodos secos y estados borrascosos desde California a Florida y Cuba, situación que persistió gran parte de la temporada de primavera (Rasmusson, 1984).

Esta manifestación en la región occidental cubana contribuye, de manera general, a que durante el invierno aparezcan con más frecuencia sistemas de bajas presiones en el área. Estos traen por consecuencia la entrada de un número mayor de frentes fríos, desajustes en la temperatura del aire y un aumento del régimen pluviométrico, el cual sin embargo disminuye en el período lluvioso. Desde el punto de vista oceánico se produce una elevación de la temperatura y del nivel del mar; esto último principalmente en la costa $\mathrm{N}$ y en repetidas ocasiones con funestas penetraciones marinas en el litoral habanero. El saldo final es negativo en varios rengiones de la economía, como en la recreación y el turismo.

Un adecuado conocimiento de los factores am bientales locales y regionales, imprescindibles para el aprovechamiento integral de los recursos naturales, no es posible sin considerar la permanente inte- racción e impacto de los procesos de escalas mayores que nos fijan el marco de referencia sobre e se desarrollan dichas condiciones (Rutllant, 1986). Por lo tanto, dada la importancia que tiene la repercusión del evento El Niño en la economía en general y en la pesca en particular, se analiza el comportamiento de algunos de los principales factores ambientales que influyen en la región occident Cuba (Fig. 1).

\section{Presión atmosférica}

El campo bárico en Cuba es generalmente dominado en invierno por el sistema de altas presiones continentales que imperan en el territorio norteamericano y que suceden a las líneas frontales con buen tiempo, regularmente frío y seco (Sosa, com. pers.). Los máximos valores promedios oscilan entre 1.017 y $1.018 \mathrm{HPa}$, mientras que los mínimos se encuen tran entre los 1.013 y 1.014 HPa (IM e IGC, 1987).

La Fig. 3 muestra las anomalías promedios de la presión atmosférica (PA), a nivel del mar en la estación meteorológica de Casablanca durante el invierno de 1923 a 1993. Nótese como la mayoría de los irregulares gradientes negativos ocurren a partir de la afectación de eventos ENOS, existiendo un período significativamente largo de anomalías negativas, que va desde 1957 hasta 1983, con algunos años de anomalías cercanas a cero y/o de anomalía positivas, como fueron las de 1966-67, 1968 y del even-

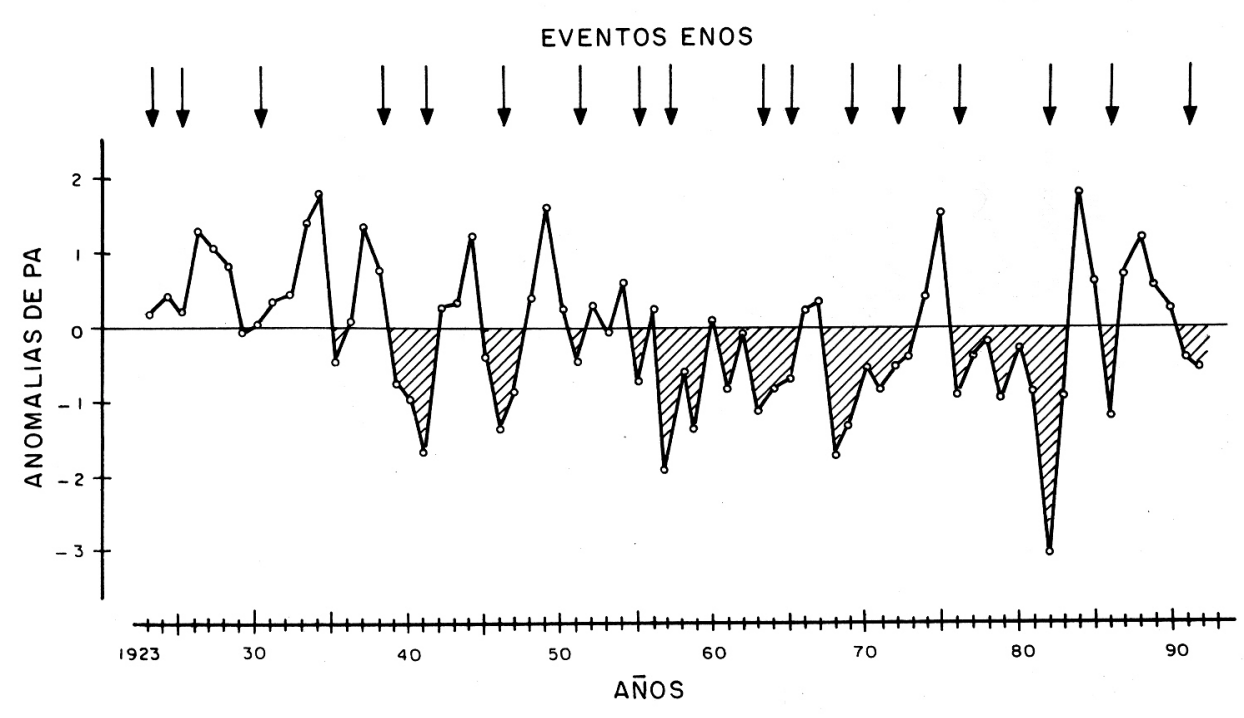

Figura 3. Anomalías de la presión atmosférica (PA) a nivel del mar en la estación meteorológica de Casablanca (promedio octubre-marzo) y años de ocurrencia de ENOS. 
to frío de 1975. El ENOS de 1982-83 afectó la presión atmosférica con registros negativos severos en todas las estaciones meteorológicas del occidente del país.

\section{Precipitaciones}

Las precipitaciones en la región occidental cubana en años de no ocurrencia de ENOS, marcan dos períodos bien definidos: uno lluvioso y otro menos lluvioso. Este último a partir de noviembre, cuando la lluvia comienza a manifestar un notable descenso (Lerch, 1984), asociado principalmente a frentes fríos con precipitaciones de corta duración. No obstante, esta sequía se muestra alterada en ocasiones por intensos acumulados de lluvia en años El Niño. Meulenert (1990) afirmó que ante eventos ENOS ocurre un aumento significativo de las precipitaciones, por encima de la media histórica en el período poco lluvioso.

La Fig. 4 muestra las anomalías promedio de las

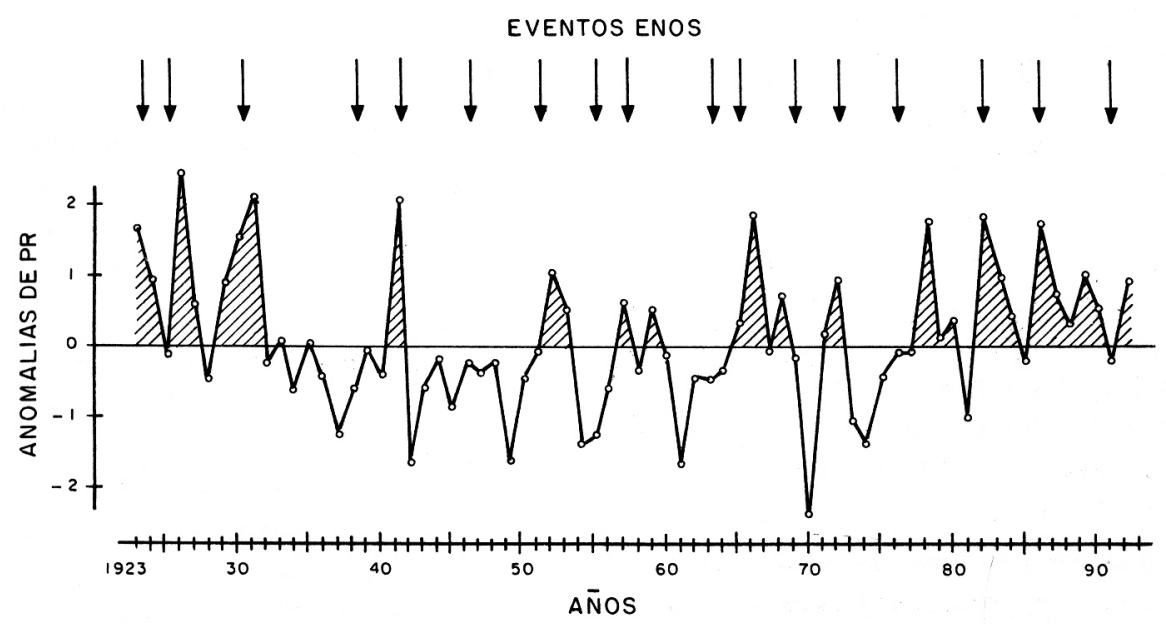

Figura 4. Anomalías de las precipitaciones (PR) en la estación meteorológica de Casablanca (promedio octubre-marzo) y años de ocurrencia de ENOS.

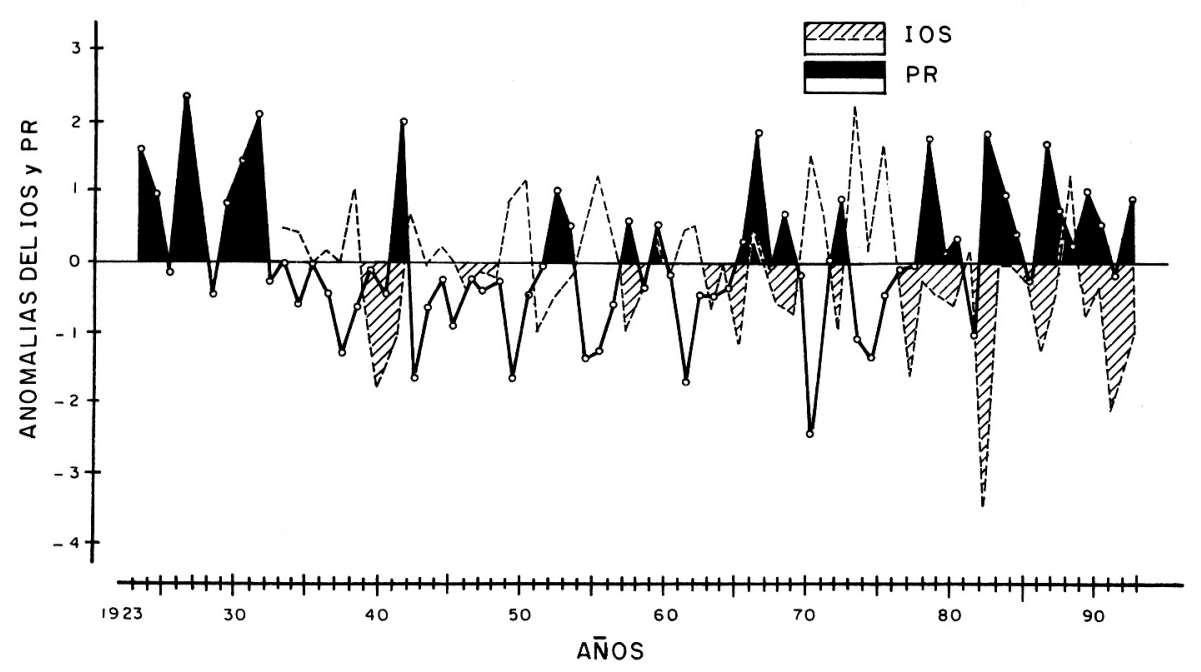

Figura 5. Anomalías del Indice de Oscilación del Sur (IOS) y de las precipitaciones (PR) en la estación meteorológica de Casablanca (promedio octubre-marzo). 
precipitaciones en la estación meteorológica de Casablanca, durante el invierno de 1923 a 1993. Se observa como a partir del invierno de 1982-83, las lluvias se han manifestado con anomalías positivas hasta 1993.

En la Fig. 5 se observa los períodos lluviosos alrededor de la fase negativa del IOS y los períodos poco lluviosos en la fase positiva. Dentro de los mecanismos más importantes que generan estas precipitaciones se encuentran: el aumento de la entrada de frentes fríos; el aumento de las anomalías de la TSM en el golfo de México y en la costa norte de Cuba; el incremento de la formación de bajas

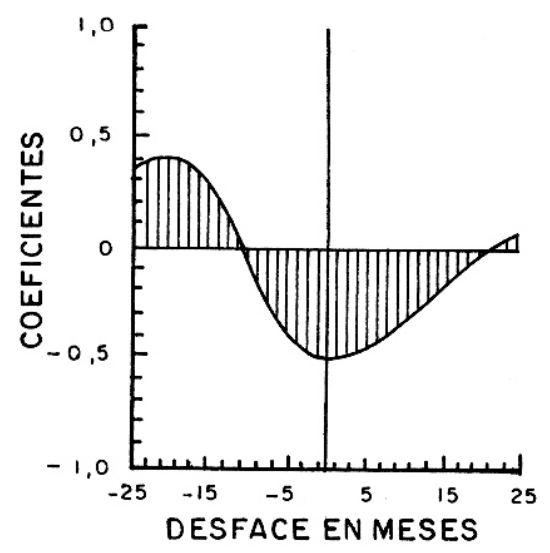

Figura 6. Coeficiente de correlación cruzada entre el IOS y las PR en la estación meteorológica de Casablaca (promedio octubre-marzo). extratropicales (Lewis y Hsu, 1992); y el traslado de altos cúmulos por la corriente en chorro subtropical, que se originan en la región Niño 3 y que trae consigo parte de la actividad convectiva que se produce en el Pacífico Ecuatorial hacia la cuenca del Caribe (Gray y Sheaffer, 1991; Hernández, 1993). El coeficiente de correlación cruzada, entre el IOS y la lluvia en Casablanca, es mayor y negativo al no considerar rezago o desfase en meses (Fig. $6)$.

\section{Temperatura del aire}

La temperatura del aire varía en invierno desde los $27^{\circ} \mathrm{C}$ como máximo promedio, hasta los $18^{\circ} \mathrm{C}$ como mínimo promedio, tomando como referencia la estación meteorológica de Casablanca (Fig. 1). La Fig. 7 muestra las anomalías promedios de la temperatura del aire (TA), en esta estación en los inviernos de 1923 a 1993 (Centella, 1991; Centella, como pers.), indicando los períodos fríos que se han manifestado en el invierno cubano. Entre ellos, los más importantes son los de la temporada 1931-32,1940, 1952 y el período comprendido entre 1966 y 1971. Los períodos cálidos que se registraron en la región occidental recayeron en las temporadas de 1946-49, 1959, 1972-75 y desde 1976 hasta nuestros días. En general, la tendencia de la temperatura del aire ante los eventos ENOS es a disminuir; sólo en los últimos cinco eventos la temperatura ha mostrado una tendencia al incremento en sus anomalías.

En la última década se ha observado una nota-

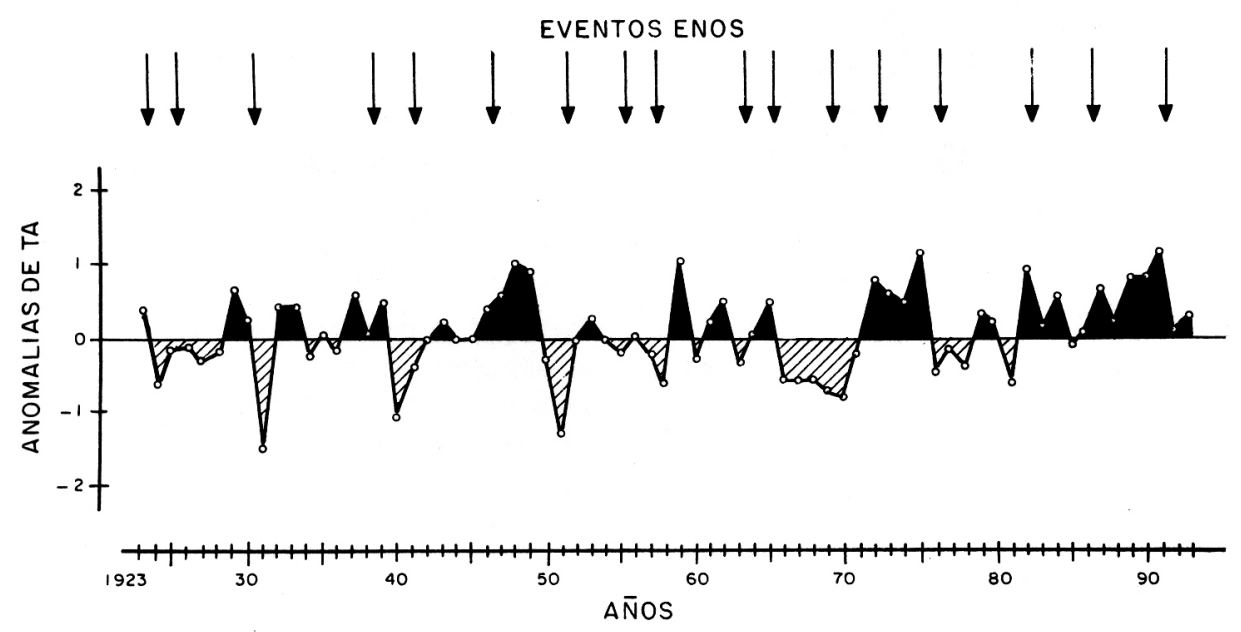

Figura 7. Anomalías de la temperatura superficial del aire (TA) en la estación meteorológica de Casablanca (promedio octubre-marzo). 


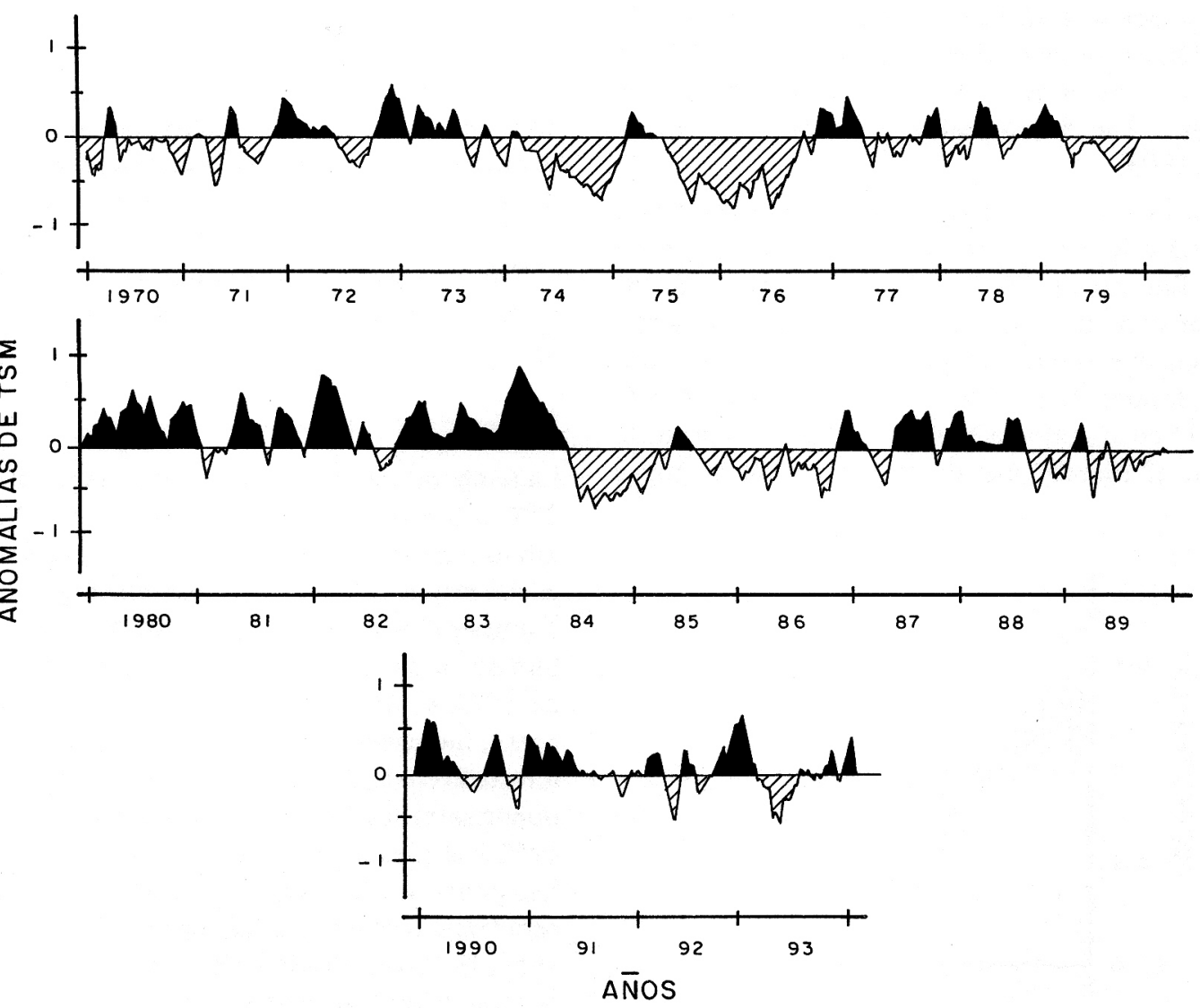

Figura 8. Anomalías mensuales de la temperatura superficial del mar (TSM) en el cuadrante de Cuba (1970-93).

ble predominancia de anomalías positivas de la temperatura media invernal en la región occidental. Esta situación es probablemente debida a que uno de los sistemas de compensación de temperatura del aire, el frente frío, ha disminuido su presencia en esta región desde su máxima expresión en la temporada 1976-77 con 35 frentes, muchos de ellos sin un contraste notable en la temperatura.

\section{Temperatura del mar}

Las primeras manifestaciones del incremento de la TSM, relacionadas con el fenómeno El Niño en la parte E del Océano Atlántico Tropical, las encontró Philander (1986) cuando constató que la capa superficial oceánica fue inusualmente caliente un año después del inicio del fenómeno El Niño en el Pacífico Sur.

A partir de la obtención por primera vez en Cuba de una serie mensual de TSM y sus anomalías re- feridas al cuadrante del país, se ha podido tener una idea del comportamiento de esta variable en el tiempo.

La Fig. 8 muestra las anomalías mensuales de la TSM desde 1970 hasta 1993. La distribución en el tiempo de las anomalías negativas muestra dos períodos significativos por debajo de la media. El primero, desde el segundo semestre de 1973 hasta diciembre de 1976, se caracterizó por presentar el mayor registro negativo de la serie en junio de 1976. El segundo período significativo de anomalías negativas, desde el segundo semestre de 1984 hasta octubre de 1986, resultó ser menos frío que el anterior y se caracterizó por ser de menor duración. La serie muestra otros años con anomalías negativas, pero al parecer éstos pertenecen a las oscilaciones propias del ciclo anual de esta variable.

Las anomalías positivas de la TSM muestran también dos períodos significativos por encima de la 


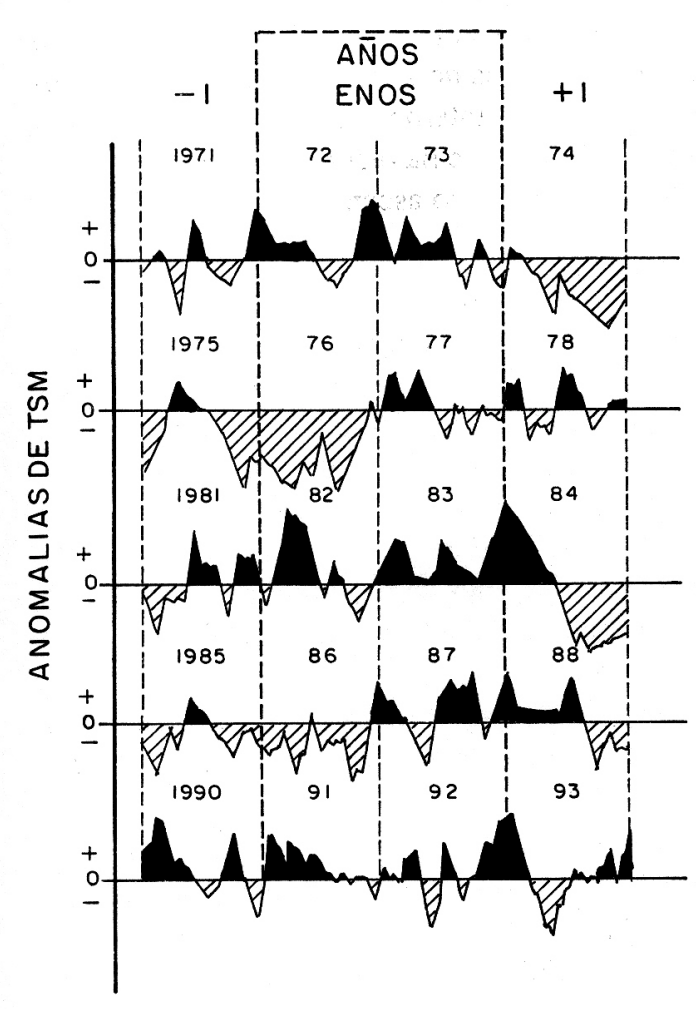

Figura 9. Anomalías de TSM en el cuadrante de Cuba durante eventos EI Niño, $y$ años pre y post evento. media. El primero desde 1980 hasta el primer semestre de 1984, siendo el más caliente, con el valor más alto reportado en diciembre de 1983 . El segundo, entre 1990 y 1993, cuyas anomalías positivas oscilan con descensos bruscos, es el período más largo de anomalías positivas.

La Fig. 9 muestra el comportamiento de las anomalías de la TSM un año antes, durante períodos El Niño y un año después del fenómeno. Se observan los incrementos de las anomalías positivas alrededor a estos años, siendo las de 1982 y 83-84 las más altas reportadas en la serie.

Durante el evento de 1972-73, después del calentamiento del primer semestre de 1972, la mayor anomalía positiva se reporta a fines de 1972 y dura hasta el segundo semestre de 1973. No sucedió así con el evento de 1976-77; en 1976 se reportaron las anomalías negativas más bajas de la serie y el calentamiento se produjo en los primeros meses de 1977. Las anomalías negativas de 1975-76, coinciden casi un año después con el período frío de 1975 en la región Niño 3 del Pacífico Ecuatorial.

Caso aparte merece el evento El Niño de 198283. Previo un intenso calentamiento que abarcó el primer semestre de 1982, las aguas presentaron nuevamente valores positivos significativos a partir de diciembre de 1982 y durante todo 1983, detectándose en diciembre de este año y en enero de 1984 los valores más altos registrados en toda la serie,

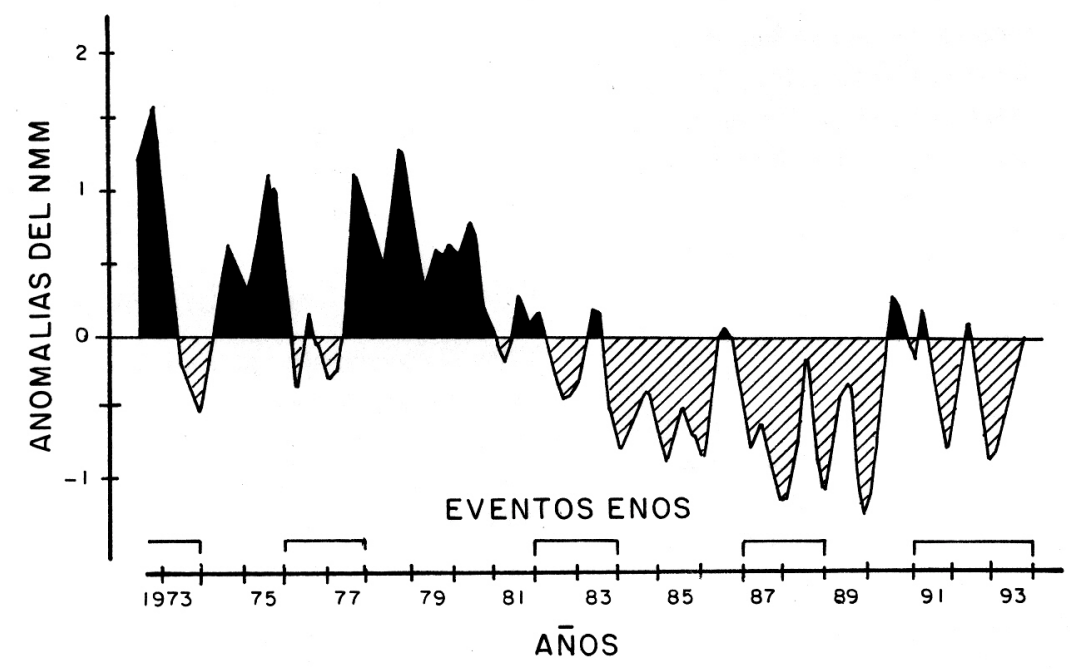

Figura 10. Anomalías del nivel medio del mar (NMM) en la estación mareográfica de Casilda. 
descendiendo posterionnente en forma notable. Las condiciones hidroclimáticas fueron severas durante el episodio, con reportes de penetraciones marinas en el litoral habanero (Rodríguez et al., 1986), escasa presencia de algunas especies marinas y reportes de especies de celenterados (medusas macroplanctónicas) de otras regiones en aguas marinas cubanas (González, como pers.).

El episodio 1986-87 fue menos intenso en lo que se refiere a la TSM. A partir de un calentamiento previo a fines de 1986 y principio de 1987, los valores de las anomalías positivas se extendieron prácticamente durante el resto de 1987 y hasta agosto de 1988.

El último evento El Niño alcanza anomalías positivas importantes hacia fines de 1992 , en enero y fines de 1993 (Fig. 9). El descenso de las anomalías de TSM a condiciones nonnales en la región Niño 3 durante la segunda mitad de 1992 (Climate Analysis Center, 1993), al parecer se reflejó en un enfriamiento de las aguas en la región de Cuba durante el segundo trimestre de 1993. Lo mismo pudiera suceder con el retorno a condiciones calientes de las anomalías de la TSM en la región Niño 3 durante el primer semestre de 1993, a la cual las aguas cubanas pudieran responder con un incremento de sus anomalías positivas durante el primer semestre de 1994, lo cual ya de hecho se manifiestó con las elevadas temperaturas en diciembre de 1993, concordando así con lo señalado por Philander (1986).

\section{Nivel medio del mar}

Son pocas las investigaciones que se han realizado sobre el NMM en Cuba. No obstante, Blázquez (1990) relacionó el ascenso del NMM en la estación Siboney, ubicada al norte de La Habana, con el efecto del ENOS de 1982-83. Los estudios de este fenómeno son aún más limitados en la costa sur del país.

Considerada como la estación cubana más con fiable en sus registros (Díaz, como pers.), la estación mareográfica de Casilda situada en el centrosur de la isla, muy cerca del golfo de Batabanó, indica que las anomalías del NMM no han sido iguales durante los últimos cinco eventos ENOS (Fig. 10).

Durante el evento de 1972-73, el NMM bajó en 1973 y se reportó alto en los dos años siguientes; en 1976-77 éste fue bajo en 1976, en 1977 comienza a ascender y el máximo se reflejó en 1978; durante 1982-83 el NMM bajó en 1982 y reflejó un bajo ascenso a mediados de 1983. A partir de este evento, que la literatura internacional señala como el más fuerte, el NMM no se ha recuperado a pesar de que en 1990 tuvo un ligero ascenso.

\section{Frentes fríos}

Los frentes fríos arriban atenuadamente por la costa noroccidental de Cuba en octubre, se incrementan de noviembre a febrero, disminuyen en marzo-abril, siendo casi nulos en mayo-junio. La Fig. 11 muestra los frentes arribados por temporada, desde 1923 hasta 1992-93. El número de frentes que arriban por temporada oscila entre 12 y 35 (consideradas como épocas extremas), siendo de vital importancia para distintas áreas de la economía del país, entre las cuales está la pesquería de langosta. donde activa el mecanismo de las migraciones masivas de este crustáceo.

Se ha observado que las temporadas invernales con mayor entrada de frentes coinciden generalmente con años donde ocurren episodios ENOS Hernández (1993) comprobó estadísticamente mediante el análisis de épocas superpuestas la correspondencia entre ambos, concluyendo que existe una asociación muy alta entre el arribo de los frentes (presión atmosférica, temperatura del aire y lluvia que le acompañan) y los eventos ENOS.

Dada la intensidad que alcanzan los frentes durante los eventos ENOS, se ha comprobado que desde 1985 a 1990 el mayor porcentaje de las penetraciones marinas que se produjeron en el litoral habanero fue generado por frentes fríos y un escaso porcentaje fue inducido por bajas extratropicales y huracanes (García et al., 1986; Rodríguez et al., 1986; Rodríguez, 1991).

\section{Huracanes y bajas extratropicales}

Otro de los eventos meteorológicos que afecta con frecuencia la región occidental de Cuba son los huracanes. Según Gray (1992), la ciclogénesis disminuye regularmente en años de influencia de ENOS; mientras que un año antes (-1) y un año después del evento $(+1)$, aumenta su frecuencia a través de la cuenca del Caribe. La Fig. 12 muestra la serie de huracanes del Atlántico, desde 1923 hasta 1993, y los años donde afectó el evento El Niño, señalando una relación casi directa en la disminución de su génesis ante este fenómeno de escala global. 
Sin embargo, la formación de bajas extratropicales en el golfo de México aumenta durante la temporada invernal asociada a períodos ENOS. Según Johnson et al. (1984), la vorticidad ciclónica en los niveles bajos de la atmósfera brinda un punto inicial para la ciclogénesis en el golfo de México, como perturbación dinámica que se mueve a través del «Jet Stream», y aseguró que las perturbaciones de la corriente en chorro u otras características en los niveles altos de la atmósfera están más relacionadas con el desarrollo de las tormentas en el área. Por otra parte, Lewis y Hsu (1992), relacionaron el incremento en la formación de estas tormentas con la frecuencia de aparición de la corriente de lazo o «Loop Current», que se manifiesta al norte de la región occidental cubana y se desplaza después por gran parte del golfo de México en forma de remolinos.

Estas tormentas generan, al igual que los frentes fríos intensos, un mecanismo de mar de leva que al topar con la costa norte de la región habanera ocasiona intensas penetraciones marinas, como las registradas en marzo de 1983, febrero de 1992 y marzo de 1993.

No cabe duda de las desventajas que para cualquier economía acompañan estas poderosas máquinas termodinámicas. Sin embargo, la influencia de ciclones en el área del golfo de Batabanó, en el período otoñal, consigue disparar el mecanismo de migraciones masivas de la langosta espinosa

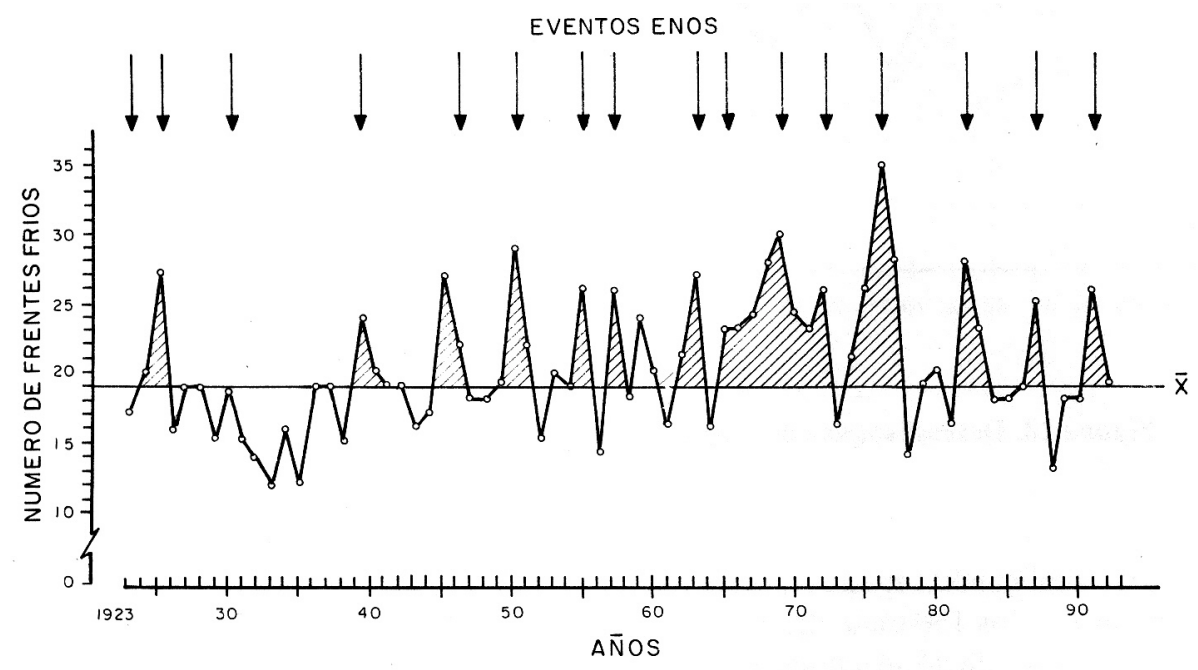

Figura 11. Frentes fríos que arribaron por la región occidental de Cuba desde 1923 hasta 1993. Observe la asociación casi directa entre éstos y los eventos ENOS.

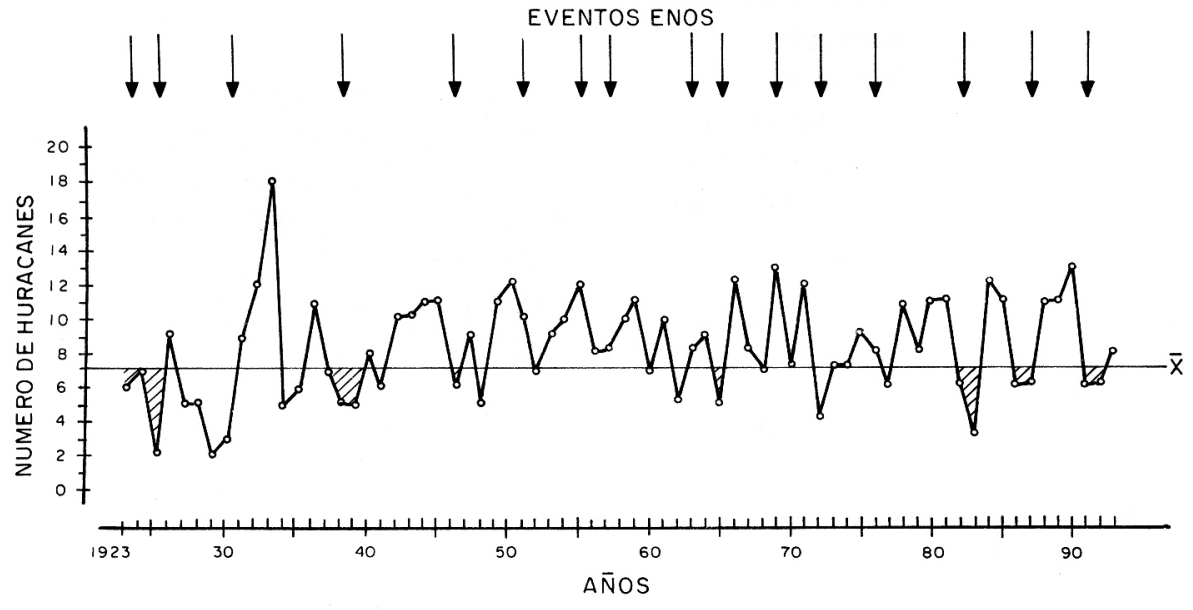

Figura 12. Actividad ciclónica del Atlántico entre 1923 y 1993. 


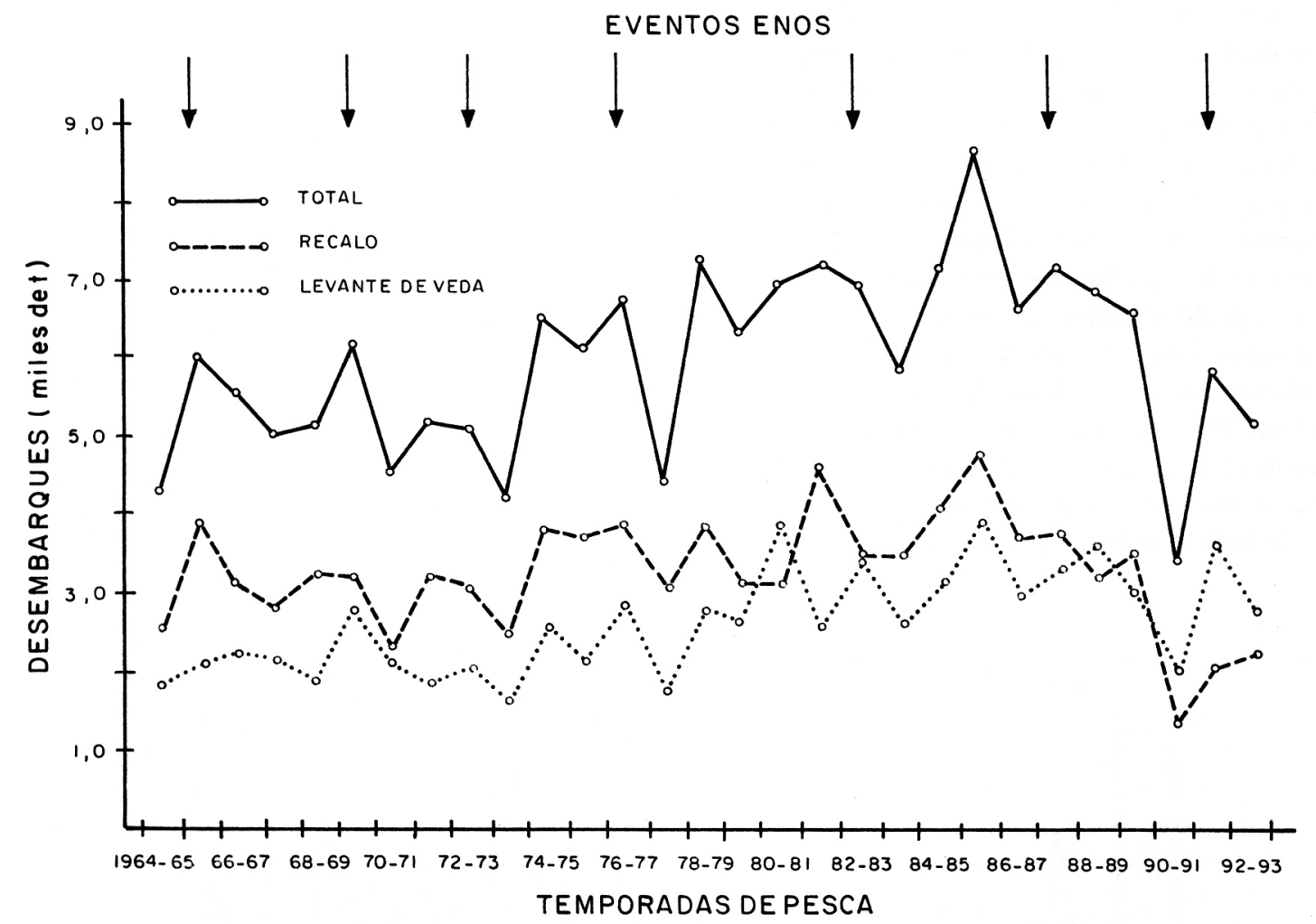

Figura 13. Desembarques de langosta (Panulirus argus) en el golfo de Batabanó.

(Panulirus argus), implicando capturas que pueden llegar a ser superiores a los 150 t/día. Tal es el caso del huracán Laura, que afectó el citado golfo en noviembre de 1971; del Katrina, que influyó también a principio de noviembre de 1981; y del Gilbert, que afectó la porción sur del golfo de Batabanó el 14 de septiembre de 1988 y provocó capturas de 144 t/día (Hernández et al., 1994). Han habido otros disturbios, como tormentas tropicales, que no se han convertido en huracanes y han originado capturas importantes, como la reportada en octubre y noviembre de 1968.

\section{Efecto de El Niño sobre la pesquería de langosta en el golfo de Batabanó.}

Muchos autores se han referido a las consecuencias que El Niño ha dejado en distintas pesquerías. Entre ellos sobresalen los trabajos de Caviedes (1975), Arntz (1984), Arntz y Arancibia (1989), Yáñez (1991) y Yáñez et al. (1994), quienes han señalado cambios en la distribución y abundancia de los re- cursos pesqueros ante la presencia de eventos ENOS. Se destaca el elaborado sobre langosta por Pearce y Philips (1988), quienes analizan los asentamientos de puerulos durante eventos ENOS y posterior pronóstico de estas pesquerías en Australia.

Arntz y Arancibia (1989), señalan que con relativa facilidad se pueden observar oscilaciones en cualquier pesquería que esté «ajustada» o no a una determinada administración pesquera. Entendiéndose por ajustada a un cierto nivel de explotación pesquera que, si bien justificada desde el punto de vista pesquero, puede ser afectada por eventos perturbantes, sincrónicos o no, que inciden en la biomasa del recurso pesquero.

En el caso de la langosta, uno de los períodos en que casi siempre se han obtenido mayores capturas es la del recalo, precisamente por la influencia directa de fenómenos perturbantes fuertes que generan o «disparan» la migración masiva, como son los frentes fríos, bajas, huracanes y diferencias significativas de la presión atmosférica. 


\section{Desembarques totales}

La Fig. 13 muestra los desembarques totales por temporada de pesca y los años de ocurrencia de eventos ENOS. En general se observa que durante los años que anteceden y preceden a estos eventos los desembarques disminuyen. Sin embargo, como se mencionó anteriormente, en noviembre de 1971 y 1981 (años pre ENOS), se produjeron capturas altas producto de la influencia en el golfo de Batabanó de los huracanes Laura y Katrina respectivamente. El desembarque más alto registrado en 1985, se atribuyó al buen reclutamiento del año anterior (Puga et al., 1992), a un alto nivel de esfuerzo (Cruz et al.,1992a), y a la influencia de cuatro ciclones tropicales en el área, dos de los cuales provocaron migraciones de langosta en el citado golfo (Hemández et al., 1994).

La Tabla 1 muestra el desembarque total, en el levante de veda y el recalo, en las temporadas de pesca de 1964-65 a 1993-94. En tanto que la Tabla 2 indica las anomalías de las capturas antes mencionadas y de los siguientes parámetros hidrometeorológicos: IOS, PA en Casablanca, TSM en la región Niño 3 y en el cuadrante de Cuba. Esta última permite comparar anomalías de variables que han sufrido alguna oscilación brusca durante los años alrededor de ENOS, entre el Pacífico y la región occidental de Cuba.

\section{Desembarques en el levante de veda}

Los desembarques registrados en el levante de veda se caracterizan por ser generalmente menores que los logrados durante el recalo (Fig. 13). No obstante, se puede apreciar que en algunas temporadas, como las de 1980-81, 1988-89 y desde 1990 hasta 1993, los desembarques en el levante superan las del recalo.

Algunos desembarques de temporadas postENOS (+1) son más elevados que los logrados durante años de afectación del evento (0), como sucedió durante la temporada de pesca de 1966-67 y 198889 (Tabla 1). Al parecer, en el primer caso la influencia del evento ENOS no había desaparecido del todo en nuestra región, como lo demuestran el IOS y la

Tabla 1. Desembarques de langosta por temporada de pesca durante años El Niño (0), y períodos pre (-1) y post (+1) evento.

\begin{tabular}{|c|c|c|c|c|}
\hline & & \multicolumn{3}{|c|}{ Desembarque (miles de t) } \\
\hline \multicolumn{2}{|c|}{ Años } & Total & Levante de & Recalo \\
\hline $\begin{array}{r}-1 \\
0 \\
+1\end{array}$ & $\begin{array}{l}64-65 \\
65-66 \\
66-67\end{array}$ & $\begin{array}{l}4,3 \\
6,0 \\
5,4\end{array}$ & $\begin{array}{l}1,8 \\
2,1 \\
2,2\end{array}$ & $\begin{array}{l}2,5 \\
3,9 \\
3,1\end{array}$ \\
\hline $\begin{array}{r}-1 \\
0 \\
+1\end{array}$ & $\begin{array}{l}68-69 \\
69-70 \\
70-71\end{array}$ & $\begin{array}{l}5,1 \\
6,1 \\
4,5\end{array}$ & $\begin{array}{l}1,9 \\
2,9 \\
2,1\end{array}$ & $\begin{array}{l}3,2 \\
3,2 \\
2,4\end{array}$ \\
\hline $\begin{array}{r}-1 \\
0 \\
+1\end{array}$ & $\begin{array}{l}71-72 \\
72-73 \\
73-74\end{array}$ & $\begin{array}{l}5,0 \\
5,0 \\
4,1\end{array}$ & $\begin{array}{l}1,8 \\
2,0 \\
1,7\end{array}$ & $\begin{array}{l}3,2 \\
3,0 \\
2,4\end{array}$ \\
\hline $\begin{array}{r}-1 \\
0 \\
+1\end{array}$ & $\begin{array}{l}75-76 \\
76-77 \\
77-78\end{array}$ & $\begin{array}{l}5,9 \\
6,7 \\
4,4\end{array}$ & $\begin{array}{l}2,1 \\
2,8 \\
1,8\end{array}$ & $\begin{array}{l}3,8 \\
3,9 \\
2,6\end{array}$ \\
\hline $\begin{array}{r}-1 \\
0 \\
+1\end{array}$ & $\begin{array}{l}81-82 \\
82-83 \\
83-84\end{array}$ & $\begin{array}{l}7,2 \\
7,0 \\
6,0\end{array}$ & $\begin{array}{l}2,5 \\
3,5 \\
2,5\end{array}$ & $\begin{array}{l}4,7 \\
3,5 \\
3,4\end{array}$ \\
\hline $\begin{array}{r}-1 \\
0 \\
+1\end{array}$ & $\begin{array}{l}86-87 \\
87-88 \\
88-89\end{array}$ & $\begin{array}{l}6,6 \\
7,1 \\
6,8\end{array}$ & $\begin{array}{l}2,9 \\
3,4 \\
3,5\end{array}$ & $\begin{array}{l}3,6 \\
3,7 \\
3,2\end{array}$ \\
\hline $\begin{array}{r}-1 \\
0 \\
+1\end{array}$ & $\begin{array}{l}90-91 \\
91-92 \\
92-93\end{array}$ & $\begin{array}{l}3,4 \\
5,6 \\
5,1\end{array}$ & $\begin{array}{l}2,0 \\
3,5 \\
2,8\end{array}$ & $\begin{array}{l}1,4 \\
2,0 \\
2,3\end{array}$ \\
\hline
\end{tabular}


Tabla 2. Anomalías de las capturas del golfo Batabanó y de variables hidrometeorológicas del Pacífico y de la región occidental de Cuba.

\begin{tabular}{|c|c|c|c|c|c|c|c|c|c|c|c|}
\hline \multirow[t]{2}{*}{ Años } & \multicolumn{3}{|c|}{ Capturas } & \multicolumn{2}{|c|}{ IOS } & \multicolumn{2}{|c|}{ PA en Casablanca } & \multicolumn{2}{|c|}{ TSM región Niño 3} & \multicolumn{2}{|c|}{ TSM cuadrante Cuba } \\
\hline & Jun-Sep & Oct-Feb & TOTAL & Abr-Sep & Oct-Mar & Abr-Sep & Oct-Mar & Jun-Sep & Oct-Feb & Jun-Sep & Oct-Feb \\
\hline $64-65$ & $-1,11$ & $-0,76$ & $-0,79$ & 0,73 & 0,08 & $-0,62$ & $-0,84$ & & & & \\
\hline $65-66^{*}$ & $-0,68$ & 1,09 & 0,32 & $-1,13$ & $-1,18$ & 0,46 & $-0,69$ & & & & \\
\hline $66-67$ & $-0,47$ & 0,07 & $-0,11$ & $-0,22$ & 0,53 & $-0,92$ & 0,20 & & & & \\
\hline $67-68$ & $-0,70$ & $-0,33$ & $-0,41$ & 0,13 & $-0,10$ & 1,10 & 0,38 & & & & \\
\hline $68-69$ & $-1,02$ & 0,18 & $-0,28$ & 0,33 & $-0,67$ & 0,46 & -1.76 & & & & \\
\hline $69-70^{*}$ & 0,51 & 0,18 & 0,37 & $-0,65$ & $-0,70$ & $-3,38$ & $-1,36$ & & & & \\
\hline $70-71$ & $-0,63$ & $-0,94$ & $-0,68$ & 0,22 & 1,50 & 0,21 & $-0,58$ & $-1,14$ & $-1,30$ & -0.04 & -0.14 \\
\hline $71-72$ & $-1,18$ & 0,22 & $-0,33$ & 0,92 & 0,58 & 0,46 & $-0,80$ & $-0,80$ & 0,76 & $-0,18$ & 0,07 \\
\hline 72-73* & $-0,86$ & $-0,06$ & $-0,35$ & $-1,35$ & $-0,93$ & $-0,23$ & $-0,53$ & 1,25 & 1,61 & $-0,26$ & 0,14 \\
\hline $73-74$ & $-1,36$ & $-0,89$ & $-0,97$ & 0,63 & 2,07 & $-0,05$ & -0.36 & $-0,91$ & $-1,38$ & $-0,07$ & $-0,11$ \\
\hline $74-75$ & 0,08 & 1,00 & 0,60 & 0,80 & 0,25 & 0,23 & 0,42 & $-0,19$ & $-0,59$ & -0.34 & $-0,38$ \\
\hline $75-76$ & -0.64 & 0,87 & 0.23 & 1.52 & 1,62 & 0.33 & 1,53 & $-1,00$ & $-1,33$ & $-0,35$ & -0.60 \\
\hline $76-77^{*}$ & 0,39 & 1,01 & 0,74 & $-0,62$ & $-0,10$ & 0,49 & $-0,96$ & 0,62 & 0,86 & $-0,55$ & 0,04 \\
\hline $77-78$ & $-1,25$ & $-0,65$ & $-0,80$ & -1.17 & $-1,52$ & 0,72 & -0.36 & 0,04 & 0.43 & -0.08 & 0.00 \\
\hline $78-79$ & 0,38 & 0.96 & 1,04 & 0,17 & $-0,25$ & $-0,36$ & $-0,18$ & $-0,47$ & 0,05 & 0.06 & 0,14 \\
\hline $79-80$ & 0.10 & 0,05 & 0,43 & 0,18 & $-0,48$ & $-1,59$ & $-0,98$ & 0,31 & 0,39 & $-0,20$ & 0,02 \\
\hline $80-81$ & 1,93 & 0,11 & 0,94 & $-0,42$ & -0.60 & $-0,56$ & $-0,29$ & 0.34 & $-0,05$ & 0.21 & 0,15 \\
\hline $81-82$ & $-0,07$ & 2,06 & 1,07 & 0,48 & 0,20 & $-0,23$ & $-0,84$ & -0.21 & 0,06 & 0,13 & 0,29 \\
\hline $82-83^{*}$ & 1,44 & $\mathbf{0 , 5 0}$ & 0,93 & $-1,48$ & $-3,09$ & $-0,79$ & $-3,09$ & 1,31 & 2,58 & $-0,03$ & 0,20 \\
\hline $83-84$ & $-0,09$ & 0,47 & 0,26 & $-0,18$ & $-0,03$ & $-0,95$ & $-0,96$ & 1,10 & $-0,05$ & 0,21 & 0,49 \\
\hline $84-85$ & 0,90 & 1,19 & 1,05 & $-0,08$ & $-0,02$ & $-0,79$ & 1,87 & $-0,68$ & $-0,80$ & 0,01 & $-0,42$ \\
\hline $85-86$ & 2,11 & 2,19 & 2,06 & 0,12 & $-0,27$ & 0,74 & 0,60 & $-0,73$ & $-0,50$ & $-0,09$ & $-0,13$ \\
\hline $86-87$ & 0,60 & 0,70 & 0,67 & $-0,18$ & $-1,27$ & 1,62 & $-1,29$ & 0,09 & 1,11 & $-0,31$ & $-0,02$ \\
\hline $87-88^{*}$ & 1,28 & 0,86 & 1,04 & $-1,62$ & $-0,42$ & $\mathbf{0 , 3 3}$ & 0,76 & 1,60 & 1,02 & 0,28 & 0,09 \\
\hline $88-89$ & 1,54 & 0,20 & 0,82 & 0,85 & 1,33 & $-1,18$ & 1,27 & $-1,47$ & $-1,28$ & $-0,04$ & $-0,12$ \\
\hline $89-90$ & 0,79 & 0,55 & 0,68 & 0,65 & $-0,72$ & 0,21 & 0,58 & $-0,23$ & $-0,11$ & $-0,06$ & 0,24 \\
\hline $90-91$ & $-0,93$ & $-2,20$ & $-1,44$ & 0,03 & $-0,33$ & 1,00 & 0,22 & 0,16 & 0,21 & 0,11 & 0,09 \\
\hline 91-92* & 1,54 & $-1,39$ & 0,03 & $-0,98$ & $-2,07$ & 0,49 & $-0,43$ & 0,83 & 1,21 & 0,06 & $-0,03$ \\
\hline $92-93$ & 0,32 & $-1,03$ & $-0,32$ & $-0,87$ & $-1,22$ & 0.57 & $-0,50$ & 0,20 & 0,05 & 0,01 & 0,24 \\
\hline
\end{tabular}

*: períodos El Niño. 
Tabla 3. Análisis de épocas superpuestas entre las capturas de langosta en el golfo de Batabanó y eventos El Niño.

\begin{tabular}{|l|l|c|c|c|c|c|c|}
\hline $\begin{array}{c}\text { Tipo de } \\
\text { análisis }\end{array}$ & $\begin{array}{c}\text { Criterio: } \\
\text { captura }\end{array}$ & $\begin{array}{c}\text { Eventos } \\
\text { clave }\end{array}$ & Intentos & $\begin{array}{c}\text { Ancho } \\
\text { epoca }\end{array}$ & $\begin{array}{c}\text { Test } \\
\text { calculado }\end{array}$ & $\begin{array}{c}\text { Intentos } \\
>\text { Test }\end{array}$ & Prob. \\
\hline $\mathrm{R}$ & Total & 6 & 10.000 & $-1,+1$ & 1,996 & 10 & 0,0010 \\
\hline $\mathrm{R}$ & $\begin{array}{l}\text { Levante } \\
\text { de veda }\end{array}$ & 6 & 10.000 & $-1,+1$ & 1,973 & 38 & 0,0038 \\
\hline $\mathrm{R}$ & Recalo & 6 & 10.000 & $-1,+1$ & 1,251 & 226 & 0,0226 \\
\hline
\end{tabular}

presión atmosférica en Casablanca (Tabla 2); mientras que en el segundo caso influyó la elevada captura por el recalo anticipado que originó la influencia del huracán Gilbert en septiembre de 1988. Los mayores desembarques se consiguieron en las temporadas 1980-81 y 1985-86, con 3,8 y 3,9 miles de t respectivamente; mientras que el mínimo se logró en la de 1973-74 con 1,6 miles de t (Fig. 13).

Los menores desembarques registrados en temporadas pre-ENOS (-1) pudieron estar influidos, entre otros, por el elevado régimen de precipitaciones y la influencia susceptible de sistemas de ondas tropicales (Sosa, 1992), bajas y huracanes que se incrementan en el Atlántico (Gray, 1992), que pueden afectar el área e impedir la pesca que se realiza en el golfo.

\section{Desembarques en el recalo}

Los desembarques se realizan de octubre a febrero y están regidos por el patrón invernal de la región occidental de Cuba. De esta manera, frentes fríos, y cualquier tormenta invernal, es capaz de generar una migración masiva de langosta a pequeña y gran escala (Baisre et al., 1985; Hernández et al, 1994).

Hernández (1990) señaló la hipótesis de trabajo acerca de la afectación de frentes y perturbaciones de tipo ciclónicas sobre las capturas de langosta en esta fase pesquera. La Fig. 13 muestra el comportamiento de los desembarques durante años de afectación del fenómeno en Cuba. El incremento de éstosen temporadas pre-ENOS (-1), como las de 197172 y 1981-82 (Tabla 1), corresponde como se señaló anteriormente a la afectación en Cuba de los huracanes Laura'y Katrina.

\section{Análisis de épocas superpuestas}

Por lo general, las investigaciones para probar rela- ciones entre eventos discretos en una serie de tiempo dada utilizan métodos estadísticos paramétricos (ej. regresión, correlación, t'estadísticos, entre otros). Estas vías implican esencialmente las siguientes suposiciones: independencia de las observaciones, normalidad, homogeneidad y/o homocedasticidad de la varianza. Los datos discretos, por su misma naturaleza, no cumplen estos requisitos, cayendo frecuentemente en us.os metodológicos inapropiados y conclusiones falsas (Prager y Hoening, 1989,1992).

La Fig. 14 muestra las anomalías de la captura total, en el levante de veda y el recalo, de las temporadas de pesca de 1964-65 hasta 1992-93, con el objeto de conocer la correspondencia entre las capturas con los años $-1,0 \mathrm{y}+1$ de ENOS, tomando como referencia el IOS y la TSM en la región Niño 3 , conformando así el análisis de épocas superpuesta.

Se asumen entonces las hipótesis nulas de cada fase pesquera para la utilización del método, como: H01: No hay asociación entre las anomalías negativas de la captura total un año antes y un año después del inicio del fenómeno.

H02: No hay asociación entre las anomalías negativas de la captura en el levante de veda un año antes y un año después del fenómeno.

H03: No hay asociación entre las anomalías negativas de la captura en el recalo un año antes y una año después del fenómeno.

Utilizando el programa SEA de Alfonzo (1992), se obtuvieron los resultados que se muestran en la Tabla 3. La asociaciones entre las capturas totales, del levante de veda y recalo, con los eventos El Niño resultan significativas, rechazándose las hipótesis nulas antes planteadas. Estos resultados demuestran 

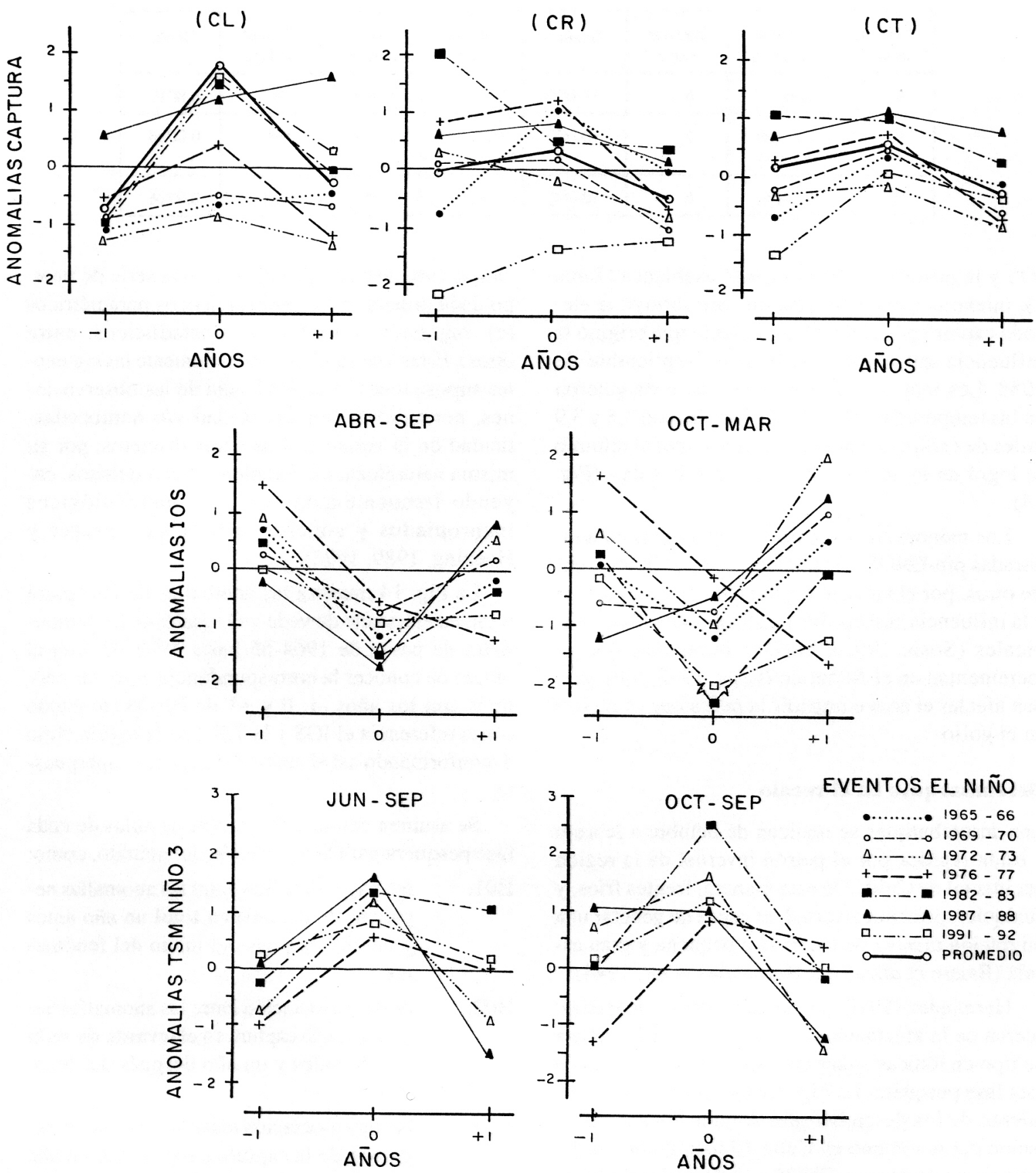

Figura 14. Conformación del análisis de épocas superpuestas: anomalías de las capturas de langosta en el levante (CL), en el recalo (CR) y totales en el golfo de Batabanó (CT), y de las referencias IOS (promedio abril-septiembre y octubre-marzo) y TSM de la región Niño 3 (promedio junio-septiembre y octubre-marzo). 

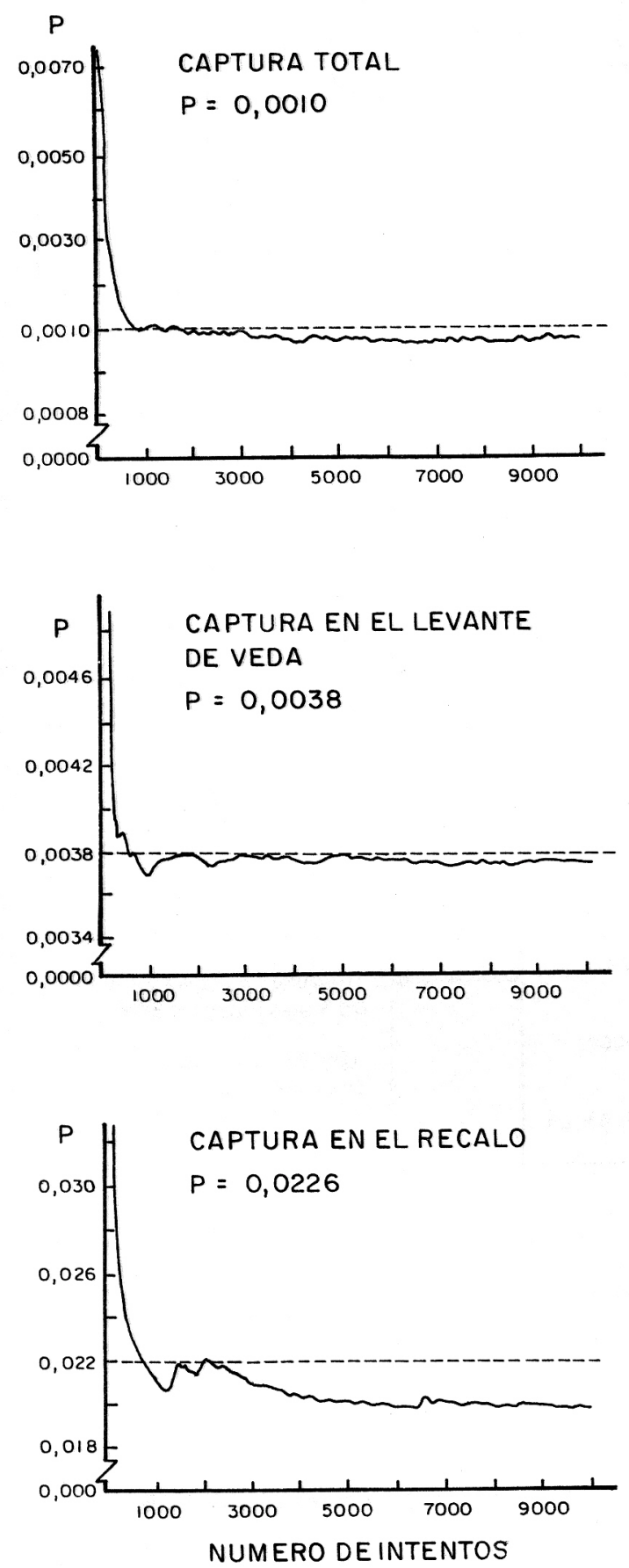

Figura 15. Nivel de significancia (P) del valor observado según la prueba $R$, como una función del número de intentos por simulación aleatoria, en la pesquería de langosta (Panulirus argus) del golfo de Batabanó. estadísticamente, el aumento de las capturas de langosta durante los años de ocurrencia de ENOS.

La probabilidad mientras más cercana al valor 0 es más dependiente del evento clave. La prueba que se obtuvo en el recalo, aunque alta, sigue siendo significativa. Como se sabe, esta fase pesquera está más relacionada con la influencia de los efectos ambientales del invierno. Sin embargo, el aumento en la formación de huracanes, justamente el año -1 y +1 del evento ENOS, provoca el aumento de las capturas un año antes y por consiguiente solapa un tanto el resultado de este análisis. Hasta el momento no se ha encontrado que un huracán haya ocasionado aumento de capturas un año después.

Estos resultados coinciden con los obtenidos por Baisre et al. (1985), Hernández (1990) y García et al. (1991), en sus estudios sobre la influencia de eventos hidrometeorológicos en las pesquerías de langosta. Cruz et al. (1992b) encontraron que existe más dependencia de la relación especie-arte (capturabilidad) con las condiciones ambientales en el recalo. La Fig. 15 muestra las probabilidades, según la simulación aleatoria por el método de Monte Carlo, en cada fase pesquera.

El modelo conceptual por el cual el fenómeno ENOS afecta la región occidental de Cuba y su incidencia posterior en la pesquería de langosta se muestra en la Fig. 16. El modelo, probablemente susceptible de ser modificado, sólo pretende dejar definido un patrón del efecto del fenómeno ENOS en la zona de estudio.

Muchas son las interrogantes que aún quedan por resolver acerca de la influencia de los eventos perturban te $\mathrm{s}$ fuertes sobre las pesquerías de especies marinas de interés comercial en las aguas cubanas. Faltarían por esclarecer las influencias del medio sobre la biología y pesquería; por ejemplo, la incidencia de la TSM sobre las hembras ovígeras, dado que es muy significativo que las mayores capturas de langosta se hayan producido casi tres años después de las mayores anomalías positivas de la TSM, coincidiendo con el tiempo de reclutamiento de esta especie a la pesquería. Otro punto a esclarecer es cómo el NMM en el sur de la isla ha descendido a partir del inicio del episodio ENOS más fuerte; o cómo la turbulencia y el transporte Ekman (Bakun, 1973) pudieran influir en la entrada, salida y supervivencia de larvas y puerulos en la interfase con el océano en la zona del golfo de Batabanó.

Sin embargo, eventos meteorológicos fuertes, 


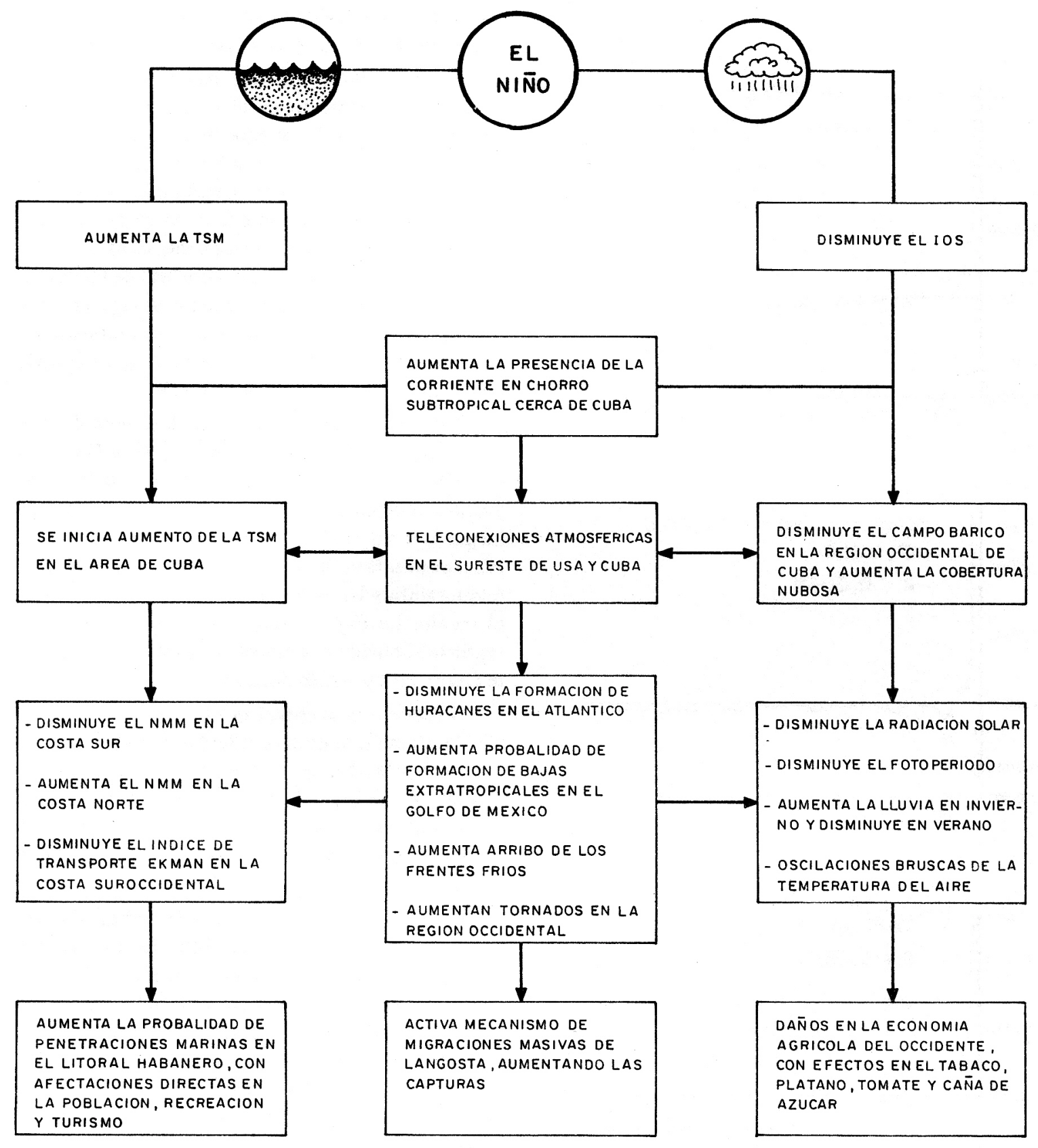

Figura 16. Modelo conceptual del impacto del fenómeno ENOS en la región occidental de Cuba.

como huracanes, frentes fríos, cambios bruscos en la presión atmosférica y ahora los fenómenos ENOS, han dejado de ser una especulación anecdótica para convertirse en hechos que, estadísticamente demostrables, afectan la pesquería de langosta.

\section{CONCLUSIONES}

Las variables hidroclimáticas, como presión atmosférica, lluvia, temperatura del aire y del mar, nivel del mar y eventos meteorológicos, como frentes fríos, 
huracanes y bajas extratropicales que suceden en la región occidental cubana, experimentan cambios relacionados con los eventos ENOS.

Se comprobó estadísticamente que las fases pesqueras de langosta en el golfo de Batabanó son favorecidas durante años de influencia de eventos ENOS, existiendo una alta probabilidad que las capturas sean bajas un año antes y un año después del evento.

\section{AGRADECIMIENTOS}

Quisiéramos dejar constancia de nuestro agradecimiento principalmente a todos los pescadores de langosta que trabajan en los distintos Combinados Pesqueros Industriales del golfo de Batabanó; a los colegas Dr. R.W. Reynolds, Dr. V. Kousky y Dr. D. Enfield por la incalculable ayuda en el suministro de datos; particularmente a los revisores de este trabajo; al Sr. P. Arana por su colaboración y apoyo; y en especial al Dr. E. Yáñez por su entusiasmo y ayuda para que este trabajo saliera publicado. A todos, muchas gracias.

\section{REFERENCIAS BIBLIOGRAFICAS}

Alfonso, A. 1992. SEA: Sistema de análisis de épocas superpuestas. Centro de Investigaciones Pesqueras de Cuba, Manual del Usuario, 9 pp.

Arntz, W.E. 1984. El Niño and Pero: positive aspects. Oceanus, 27 (2).

Arntz, W.E. y H. Arancibia. 1989. Fluctuaciones en el eco sistema demersal del Pacífico SurOriental y el Norte de Europa: una comparación. Pacífico Sur, Número Especial: 35-47.

Baisre, J., C. García y R. Cruz. 1985. Migraciones masivas de la langosta ( $P$. argus) en la plataforma cubana. Centro de Investigaciones Pesqueras de Cuba. In litteris.

Bakun, A. 1973. Coastal upwelling indices, west coast of North America, 1946-71. U.S. Dep. Commer., NOAA Tech. Rep. NMFS/SSRF 671,103 pp.

Blázquez, L. 1990. Anomalías del nivel del mar en La Habana-Cuba durante el evento «Oscilación del Sur-El Niño»(OSEN) de 1982-83. ACC, Rep. Inv., 6.
BMA. 1992. Darwin Tropical Diagnostic Statement. BureauofMeteorologyinAustralia(BMA), 11 (7).

Bradley, R.S., H.F. Díaz, G.N. Kiladis y J.K. Eischeid. 1987. ENSO, signal in continental temperature and precipitation records. Nature, 327: 497-501.

Cane, M.A., S.E. Zebiak y S.C. Dolan. 1986. Experimental forecast of El Niño. Nature, 321.

Caviedes, C.N. 1975. El Niño 1972: It's climatic, ecological, human, and economic implications. Geog. Rev., 65: 493-509.

Centella, A. 1991. La temperatura del aire en la estación de Casablanca. I Taller sobre Cambios Climáticos y sus Consecuencias. ACC, La Habana, Cuba.

Climate Analysis Center. 1993. Climate Diagnostic Bulletin. U.S. Dep. Commer., NOAA, 1-12.

Cruz, R., R. Sotomayor, M.E. de León y R. Puga. 1992a. Impacto del manejo de la pesquería de langosta ( $P$. argus) en el archipiélago cubano. Rev. Inv. Mar., 12 (1-3).

Cruz, R., R. Puga y M.E. de León. 1992b. Aspecto de la dinámica de poblaciones de la langosta espinosa ( $P$. argus) en Cuba: cambios en el coeficiente de capturabilidad. Centro de Investigaciones Pesqueras de Cuba, Res. I Sem. Cienc. y Técn., 16-17 Enero.

Dickson, R.R. y J. Namías. 1976. North American influences on the circulation and climate of the North Atlantic sector. Mont. Wea. Rew., 104: 12561265.

Enfield, D.B. 1987. Progress in understanding El Niño. Endev. New. Serv., 11 (4).

Galindo, I. y G. Zenteno. 1989. Peculiaridades del fenómeno «El Niño-Oscilación Suriana» en la porción noroeste tropical del Océano Pacífico. Como Téc. Serv. Inv., UNAM, 100: 1-4.

García, O., C. González, A. Meulenert y J.D. Arias. 1986. Análisis de las penetraciones del mar en las costas cubanas durante 1985. ACC, Instituto de Meteorología, La Habana, Cuba.

García, C., B. Hernández, J. Baisre y R. Cruz. 1991. Factores climáticos en las pesquerías cubanas de langosta $(P$. argus $)$ : su relación con las migraciones masivas. Rev. Inv. Marin., 12 (1-3). 
Glantz, M.H. 1991. Introduction. In: Teleconnection Link:ing Worldwide Climate Anomalies. M. Glantz, R. Katz and N. Nicholls (Eds.), Cambridge University Press, New York: 1-12.

Gray, W.M. y J.D. SheatTer. 1991. El Niño and QBO influences on tropical cyclone activity. In: Teleconnection Linking Worldwide Climate Anomalies. M. Glantz, R. Katz and N. Nicholls (Eds.), Cambridge University Press, New York: 257-284.

Gray, W.M. 1992. Update forescal of Atlantic seasonal hurricane activity for 1992. Clim. Diag. Bull., 7: 44-45.

Haurtwizt, M. y G.W. Brier. 1981. A critique of the Superposed Epoch Analysis method: It's application to solar-weather relations. Mon. Wea. Rev., 109: 2074-2079.

Hernández, B. 1990. Recalo de la langosta en 198687. Rev. Cubo Inv. Pesq., 15 (1-4).

Hernández, B. 1992. Análisis hidrometeorológico de las plataformas cubanas en 1991. Centro de Investigaciones Pesqueras. In litteris.

Hernández, B. 1993. «El Niño», los frentes fríos y algunas señales del ENOS en Cuba. Centro de Investigaciones Pesqueras de Cuba. In litteris.

Hernández, B., C. García y J. Baisre. 1994. ¿Pueden los ciclones tropicales provocar migraciones masivas de langosta? Proceeding VIII Congreso Brasilero e Iberoamericano de Meteorologia, Sao Pablo, Brasil.

Hoening, J.M., M.H. Prager y N.B. Payton. 1989. Computer programs for investigating the effects of environmental events on a time series of recruitment. Can. Tech. Rep. Fish.Aqua. Sci., 1713: $1-5$.

Horel, J.D. y J.M. Wallace. 1981. Planetary scale atmospheric phenomena associated with the Southem Oscillation. Mon. Wea. Rew., 109: 813829.

IM e IGC. 1987. Atlas Climático de Cuba. Instituto de Meteorología (IM) e Instituto de Geodesia y Cartografía (IGC), La Hahana, Cuba.

Johnson, G.A., E.A. Meindl, E.B. Mortimer y J.S.
Lynch. 1984. Features associated with repeated strong cyclogenesis in the westem Gulf of Mexico during the winter of 1982-83. Am. Met. Soc.: $110-117$.

Lerch, G. 1984. Diagramas climáticos de Cuba. ACC, La Habana, 12 pp.

Lewis, J.K. y S.A. Hsu. 1992. Mesoescale airsea interactions related to tropical and extratropical storm in the Gulf of Mexico. J. Geophy. Res., 97 (2): 2215-2228.

Meehl, G.A. 1978. Tropical teleconections to the seesaw in winter temperatures between Grenland and Northen Europe. In: Teleconnection Linking Worldwide Climate Anomalies. M. Glantz, R. Katz and N. Nicholls (Eds.), Cambridge University Press, New York: 429-492.

Meulenert, A. 1990. Efecto del evento «El Niño/ Oscilación del Sur» sobre el estado del tiempo en Cuba. Instituto de Meteorología, La Habana, Cuba. In litteris.

Nicholls, N. 1986. A method for predicting Murray Valley Encephalitis in Southeast Australia using SO. Aust. J. Exp. Biol. and Med. Sc., 64: $587-594$

Panofski, W. y G.W. Brier. 1965. Some applications of statistics to meteorology. Coll. Min. Ind., Pennsylvania State Univ., 159 pp.

Pauly, D. 1987. Managing the Peruvian upwelling ecosystem: a sinthesis. In: The Peruvian anchoveta ant its upwelling ecosystem: three decades of change. D. Pauly and I.Tsukayama (Eds.), ICLARM Studies and Reviews, 15: 325-342.

Pearce, A. y B. Philips. 1988. ENSO events, the Leewin current, and larval recruitment of the westem rock lobster. J. Cons. int. Exp. Mer, 44: 18-26.

Philander, S.G.H. 1986. Unusual conditions in the tropical Atlantic Ocean in 1984. Nature, 322: 236238.

Prager, M.H. y J.M. Hoening. 1989. Superposed epoch analysis: a randomization test of environmental effects on recruitment with application to chub mackerel. Trans. Amer. Fish. Soc., 118: 608-618.

Prager, M.H. y J.M. Hoening. 1992. Can we determine the significance of the key-event 
effects on a recruitment time series?: a power study of Superposed Epoch Analysis. Trans. Amer. Fis. Soc., 121(1): 123-131.

Puga, R., R. Cruz y M.E. de León. 1992. Tendencias del reclutamiento a la pesquería de la langosta $(P$. argus $)$ en el golfo de Batabanó. Centro de Investigaciones Pesqueras de Cuba, Res. I Sem. Cienc. y Tec. Pesq., 16-17 Enero.

Quinn, W. y V.T. Neal. 1989. The historical record of El Niño events. In: Climate since A.D. 1500. Bradley \& Jones, London.

Rasmusson, E.M. 1984. El Niño: The Ocean/ Atmosphere Connection. Oceanus, 27 (2): 5-12.

Rasmusson, E.M. 1991. Observational aspects of ENSO cycle teleconnection. In: Teleconnection Linking Worldwide Climate Anomalies. M. Glantz, R. Katz and N. Nicholls (Eds.), Cambridge University Press, New York: 309-343.

Rasmusson, E.M. y J. Wallace. 1983. Meteorological aspects of El Niño/Southem Oscillation. Science, 222: 1195-1202.

Rodríguez, M. 1982. Cronología de los frentes fríos desde 1916 hasta 1982. Instituto de Meteorología de Cuba, Contribución $\mathrm{N}^{\circ} 9$.

Rodríguez, E. 1991. Cronología y análisis sinóptico de las penetraciones marinas en Ciudad de La Habana (1986-1990), con referencia a una escala de intensidad. Instituto de Meteorología de Cuba. In litteris.

Rodríguez, E., I. Guerra y J.C. Cárdenas. 1986. Cronología y análisis sinóptico de las penetraciones del mar en las provincias habaneras 19701986. I Cong. Int. sobre Desastres Nat., La Habana, Cuba.

Ropelewski, C.F. y M.S. Halpert. 1986. North American precipitation and temperature pattems associated with the El Niño/Southem Oscillation (ENSO). Mont. Wea. Rew., 114: 2352-2362.

Rutllant, J. 1986. Interacción océano-atmósfera. Rev. Geofísica, Univ. de Chile, 24.

Sosa, M. 1992. Cronología de las ondas tropicales en Cuba. Tesis de Doctorado: TI Parte. Instituto de Meteorología, La Habana, Cuba.

Sotomayor, R. y R. Cruz. 1991. SISLAN: Sistema para la evaluación y administración de la pesquería de langosta. Centro de Investigaciones Pesqueras de Cuba, Catálogo de Software.

Trenberth, K.E. 1991. General characteristics of El Niño-Southem Oscillations. In: Teleconnection Linking Worldwide Climate Anomalies. M. Glantz, R. Katz and N. Nicholls (Eds.), Cambridge University Press, New York: 13-42.

van Loon, H. y J.C. Rogers. 1978. The seesaw in winter temperatures between Greenland and Northern Europe. Part I. General Description. Mont. Wea. Rew., 108(3): 296-310.

White, M.E. y M.W. Downton. 1991. The shrimp fishery in the Gulf of Mexico: relation to climate variability and global atmospheric pattems. In: Teleconnection Linking Worldwide Climate Anomalies. M. Glantz, R. Katz and N. Nicholls (Eds.), Cambridge University Press, New York: 429-492.

Yáñez, E. 1989. Fluctuaciones de los principales recursos pelágicos explotados en la zona norte de Chile y variaciones ambientales asociadas. Pacífico Sur, Número Especial: 509-520.

Yáñez, E. 1991. Relationships between environmental changes and fluctuating major pelagic resources exploited in Chile (1950-1988). In: Long- Term Variability of Pelagic Fish Populations and their Environment. T. Kawasaki, S. Tanaka, T. Toba and A. Taniguchi (Eds.), Pergamon Press: 301-309.

Yáñez, E., M.A. Barbieri, A. González, O. Pizarro, M. Bravo, C. Canales y S. Salinas. 1994. Sea surface thermal structure associated to the pelagic fish resources distribution in northem Chile. In: Remote Sensing for Marine and Coastal Environments: Needs, Solutions and Applications. ERIM, Michigan, USA, Vol. TI: 149-162.

Zebiak, S.E. y M.A. Cane. 1987. A model El NiñoSouthem Oscillations. Mon. Wea. Rev.. 115: 2262-2278. 
